\title{
Aspekte einer Anthropologie des Fernsehzuschauers
}

\author{
von Rainald Merkert
}

In jüngster Zeit ist von verschiedenen Autoren eine gewisse Sterilität der Medienwirkungsforschung konstatiert worden. Andere sprechen vom Stagnieren dieser Forschung. Damit ist nicht gemeint, daß heute weniger geforscht wird, sondern da $B$ man trotz aller Forschung im Grunde kaum weiterkommt. Einerseits gibt es inzwischen eine kaum noch überschaubare Fülle von Einzeluntersuchungen: „Wie mit dieser Fülle fertig werden? Wer von uns vermöchte auch nur zu sagen, er überblicke die wichtigsten Arbeiten zum Bereich der kognitiven, der sozialen oder emotionalen Medienwirkungen?", so klagt etwa H. Sturm ${ }^{1}$. Andererseits sind die vorliegenden Ergebnisse trotzdem spärlich, punktuell, heterogen. Kaum eine einschlägige Publikation unterläßt den Hinweis darauf, daß die meisten Aussagen über Medienwirkungen noch vorläufigen hypothetischen Charakter haben, daß die Medienforschung bisher mehr Defizite als Ergebnisse vorzuweisen habe. Trotz der Forschungsfülle gebe es so gut wie keine gesicherten Erkenntnisse über allgemeine $\mathrm{Zu}$ sammenhänge in der Empirie der Wirkungsprozesse, im Gegenteil: „Methodologisch bildet ein Grundproblem dieses ganzen Forschungsbereichs, daß regelmäßig detailliertere Untersuchungsanlagen die Generalisierungen der vorangehenden Arbeiten dementieren. ${ }^{22}$

Diese Situation ist für alle Bereiche der Mediennutzung charakteristisch. Als zum Beispiel das Sekretariat der Kultusministerkonferenz im Jahre 1974 eine Umfrage bei allen Kultusbehörden nach Untersuchungen zum öffentlichen Schulfernsehen durchführte, wurden 50 Arbeiten gemeldet. Als G. Tulodziecki drei Jahre später „Eine Zusammenstellung von Ergebnissen aus Begleituntersuchungen zu Projekten öffentlichen Schulfernsehens" herausgab, basierte seine Arbeit auf insgesamt 74 Forschungsberichten. Doch die Quantität der Untersuchungen steht offenbar selbst in diesem speziellen Bereich im Mißverhältnis zu ihrer Qualität, obwohl sich doch der in didaktischer Absicht erfolgende Medieneinsatz ungleich präziser untersuchen läßt als die Wirkungen des allgemeinen Fernsehprogramms. Jedenfalls ist Tulodzieckis Resümee deprimierend: Die vorliegenden Ergebnisse seien mehr oder weniger nichtssagend, weil ihnen keine "wohldefinierten Hypothesen" zugrundelägen; sie seien eher „Deskriptionen des Untersuchungsfeldes“, „nur erste Antworten auf Fragen ${ }^{\text {‘3 }}$. Die aus ihnen abgeleiteten Forderungen für die Gestaltung von Schulfernsehsendungen seien folglich ,lediglich subjektive, aus dem situativen Gesamtzusammenhang resultierende Meinungen ${ }^{\text {"4 }}$.

Die Gründe für diese mißliche Situation der Wirkungsforschung, die freilich mitunter auch negativer dargestellt wird als sie ist, sind unterschiedlicher Art. Wenigstens einige sollen genannt werden. Einmal ergeben sich die untersuchten Fragestellungen selten aus dem Fortgang der Wissenschaft, häufiger werden sie von außen an sie herangetragen. „Unterschiedliche Auftraggeber vergeben an unterschiedliche Institutionen und damit an unterschiedliche wissenschaftliche Disziplinen eine Reihe von unterschiedlichen Forschungsaufträgen mit unterschiedJichen Intentionen und somit auch unterschiedlichen Resultaten!" In dieser

Dr. Rainald Merkert ist Akademischer Oberrat am Erziehungswissenschaftlichen Institut der Universität Düsseldorf. 
grimmig-ironischen Formulierung J. Hackforths ${ }^{5}$ ist zugleich schon ein zweiter Grund genannt. Es sind unterschiedliche wissenschaftliche Disziplinen mit ihren spezifischen Fragestellungen, die sich mit dem Einfluß der Medien befassen: Psychologie und Soziologie, Publizistik- und Kommunikationswissenschaft, aber auch Linguistik und Ästhetik, Politologie und Philosophie, Jura und Betriebswirtschaft, Erziehungswissenschaft und Kulturwissenschaften. Und innerhalb dieser Disziplinen gibt es wiederum jeweils unterschiedliche konkurrierende Schulen; ihre Ansätze sind teils spezifisch für eine Wissenschaft, teils mehrere Wissenschaften übergreifend. Diese Vielfalt der Ansätze bringt mit sich, daß auch die Terminologie nicht einheitlich ist.

Ein entscheidender Grund liegt in der untersuchten Sache selbst. Man weiß heute, daß es keine oder doch fast keine monokausalen, linearen Medienwirkungen gibt. Diese sind vielmehr eingebettet in ein komplexes Bedingungsgefüge, sie hängen von einer Vielzahl von interdependenten Variablen $a b$. Neben individuellen, gesellschaftlichen und kulturellen spielen auch situative Faktoren eine Rolle; neben den Wirkungen der Medien, von denen das Fernsehen wiederum nur eines ist, wenn auch das wichtigste, stehen die Wirkungen anderer informierender, belehrender, meinungsbildender Faktoren. Oder richtiger gesagt: nicht neben ihnen, sondern in Wechselwirkung mit ihnen; denn die Wechselwirkung macht ja gerade das Problem aus. So führt es letzten Endes nicht weiter, wenn wie bisher vorwiegend ,atomistisch Typen von Einstellungsänderungen und andere Kommunikationseffekte" untersucht werden ${ }^{6}$. Vor allem ist so den wichtigsten, nämlich den langfristigen Wirkungen nicht beizukommen.

Die Einsicht in dieses komplexe Bedingungs- bzw. Wechselwirkungsgeflecht hat neuerdings insbesondere jüngere Autoren zu grundsätzlicher Skepsis gegenüber der bisherigen „empirischen Medienwissenschaft“ veranlaßt. „Wirkungsforschung ja, aber anders!", lautet ihre Devise. Sie fordern neue adäquatere Forschungs- bzw. Theorieansätze, neue Methoden und Perspektiven der Forschung, einen Wechsel der "Paradigmen"7 - wobei freilich die modische UUbernahme des ParadigmaBegriffs von dem spezifischen Kontext absieht, in dem Th. Kuhn ihn geprägt hat ${ }^{8}$. Das bloße behavioristische Stimulus-Response-Modell soll als unzureichend überwunden werden. Die einen favorisieren den Nutzen/Belohnungsansatz (uses and gratification approach), der sich zumeist an der Theorie des symbolischen Interaktionismus orientiert ${ }^{9}$; andere bringen neuerdings die - primär bildungs- bzw. medienpolitisch orientierte - Theorie von der wachsenden Wissenskluft zwischen den Bevölkerungsschichten durch die Massenmedien in die Diskussion ${ }^{10}$; wieder andere sind noch dabei, anknüpfend an vorhandene "theoretische Vorarbeiten"den „allein weiterhelfenden“ Ansatz zu konstruieren ${ }^{11}$.

Aus der Sicht des Nutzenansatzes erscheint der Zuschauer nicht mehr als bloßes Objekt, auf den das Fernsehen einwirkt (der Begriff der Wirkungsforschung entstammt ja ursprünglich diesem Denkmodell), sondern als Subjekt, das eigentätig, also aktiv das Fernsehen rezipiert, es dabei nach seinen „Bedürfnissen“ nutzt und interpretiert. Freilich erhebt sich dann sogleich die Frage, woher denn die Bedürfnisse kommen, welches falsche und „wahre" Bedürfnisse oder, um mit Rousseau zu sprechen, welches natürliche und nur eingebildete Bedürfnisse sind. Die Frage stellt sich zwar nicht einer Wissenschaft, die sich nur als analysierendes System versteht, trotzdem ist sie unumgänglich. Prompt weisen Kritiker des Nutzenansatzes 
darauf hin, daß er fälschlicherweise „glaubt, auf eine Theorie der menschlichen Bedürfnisse verzichten zu können“. Für sie als Vertreter der Sozialwissenschaften gilt, daß „soziale Strukturen und Interaktionen die ontogenetische Entwicklung konstituieren“, also mehr sind als bloß kontingente Randbedingungen einer „bislang immer psychologisch begriffenen Ontogenese" ${ }^{\text {12. }}$.

Doch soll hier nicht näher auf diese oder ähnliche Kontroversen eingegangen werden. Sie sind häufig auch deshalb unfruchtbar, weil die Autoren die jeweils andere Position mit Nachdruck, wenn nicht sogar überheblich kritisieren, ihre eigenen Ansätze aber keineswegs immer überzeugender und, wie etwa der Nutzenansatz, noch seltener neu sind ${ }^{13}$. Hier soll nur der gegenwärtige Trend festgehalten werden, ein weiteres Auseinanderlaufen unterschiedlicher Forschungstraditionen, eine fortschreitende Anhäufung mehr oder minder isolierter Einzeldaten zu überwinden durch eher integrative Theorieansätze bzw. durch Theorien von größerer Reichweite. Nicht nur neue Modelle oder Paradigmen werden gesucht, sondern der komplexen Kommunikationswirklichkeit adäquatere Modelle. J. R. Brown fordert sogar ausdrücklich „einen konzeptionellen Rahmen“, der zum einen „die verschiedenen Untersuchungen zusammenbringt", zum anderen ,auch neue und wichtige Fragen für die Forschung aufzeigt" ${ }^{\text {*14 }}$. Im folgenden soll es nur um eine solche umgreifende Rahmentheorie gehen.

\section{Die Frage nach einem integrativen Rabmen}

Brown meint im Hinblick auf das Fernsehen der Kinder, „ein solcher integrativer Rahmen kann durch eine Untersuchung der kindlichen Fernsehnutzung im Kontext der kindlichen Sozialisation entwickelt werden" ${ }^{\text {"15 }}$. Er skizziert einige der sozialen Situationen, ,in die das Fernsehen als Träger von Sozialisationsbotschaften eingebettet ist“, und fragt dann, ob nicht beispielsweise „kontrollierte Laboruntersuchungen über die Wirkung eines 10minütigen Anschauens von Gewaltsendungen unendlich weit von der Vorstellung der 'realen Welt' entfernt sind, wie sie sich sowohl Wissenschaftler wie Laien nützlicherweise zurechtgelegt haben“. Nach seiner Meinung ist das jedoch nicht der Fall, denn eine solche empirische Untersuchung könne „im Gesamtbezugsrahmen angesiedelt" sein und auf diese Weise eher auf andere Forschungsschwerpunkte bezogen werden ${ }^{16}$.

Brown schließt sich also der gegenwärtig vorherrschenden Sozialisationstheorie bzw. Sozialisationsforschung an, die das Fernsehen als Faktor im Sozialisationsprozeß begreift. Die Genese des Menschen wird hier nicht mehr in erster Linie als Entwicklung, sondern als Vergesellschaftung verstanden. Kulturanthropologen sind mit diesem Ansatz nicht einverstanden, für sie ist es nicht die Gesellschaftlichkeit, die den Menschen ausmacht, sondern die Kultur. Auch die höheren Tiere leben gesellschaftlich $^{17}$; was das soziale Wesen Mensch dennoch vom sozialen Wesen Tier unterscheidet, ist die Tatsache, daß dem Menschen die konkreten Formen des Zusammenlebens nicht angeboren sind wie dem Tier, daß er sie vielmehr selbst schaffen muß, daß sie also das Ergebnis von „Kultur“ sind. Wenn aber der Mensch primär Kulturwesen ist, dann erscheint seine Genese konsequenterweise in erster Linie als Enkulturation und erst in zweiter Linie als Sozialisation. Und entsprechend wäre das Fernsehen dann als Enkulturationsfaktor zu fassen. Nun soll diese Position, so interessant sie ist, hier nicht vertreten werden, schon aus pragmatischen Gründen nicht; sie wird nur erwähnt, um darauf hinzuweisen, daß 
auch der Sozialisationsbegriff durchaus nicht ohne anthropologische Implikationen ist.

Orientiert man sich nun am Sozialisationsbegriff, so darf man doch nicht übersehen, daß er keineswegs eindeutig ist. Sozialisation kann, wie bei T. Parsons, affirmativ oder anpassungsmechanistisch verstanden werden. Dann aber macht der homo sociologicus nicht den ganzen Menschen aus, wie $R$. Dahrendorf betont ${ }^{18}$, die Sozialisation also auch nicht den Gesamtprozeß der Menschwerdung, neben ihr steht vielmehr gleichrangig die Individualisation. Man kann Sozialisation aber auch umfassender verstehen, wie es heute zumeist geschieht. So betont etwa J. Kob, daß „auch und gerade in einer soziologischen Sozialisationstheorie die Autonomie der Person ganz grundsätzlich und von vornherein als Gesichtspunkt mitaufzunehmen “sei; „die Aneignung von Regelhaftigkeiten, Orientierungsstilen, Wertungen einer bestimmten Gesellschaft und die damit vollzogene Eingrenzung der prinzipiell enorm weiten Spanne der eigenen Möglichkeiten auf eine bestimmte Identität der Person sind grundsätzlich ebenso sehr als Handlungen des Individuums wie als Erleiden differenzierter sozialer Zwänge zu denken “19. Wenn aber der Sozialisationsbegriff so weit gefaßt wird, dann ist nicht einzusehen, warum der von ihm gemeinte Prozeß nicht genau so richtig als „Personalisation“ bezeichnet werden kann. Doch soll auch das hier nicht diskutiert, sondern nur angemerkt werden, um nochmals darauf hinzuweisen, daß eine so verstandene Theorie der Sozialisation voller anthropologischer Implikationen ist, ja bereits in die Nähe einer anthropologischen Theorie gelangt.

Hier soll vielmehr versucht werden, den weiteren Schritt auf eine Anthropologie des Fernsehzuschauers hin zu tun. Die von Brown vertretene These, der gesuchte integrative Rahmen sei der Sozialisation abzugewinnen, steht nämlich vor einer weiteren Schwierigkeit, die mit den angedeuteten anthropologischen Implikationen zusammenhängt: es gibt (noch) keine umfassende Gesamttheorie der Sozialisation, sondern nur konkurrierende Teiltheorien. Sie orientieren sich zum einen an verschiedenen Wissenschaftsrichtungen, gehen zum anderen von unterschiedlichen Forschungsinteressen aus. In den einschlägigen Darstellungen werden zumeist genannt: der lerntheoretische oder behavioristische Ansatz, der psychoanalytische Ansatz, der rollentheoretische Ansatz, der Ansatz des symbolischen Interaktionismus und der des Kognitivismus.

Bevor wir diesen weiteren Schritt versuchen, ist jedoch noch auf einen anderen Typ von Theorien hinzuweisen, die einen „konzeptionellen Bezugsrahmen für die Entwicklung begrifflich integrierbarer Teiltheorien und operationalisierbarer Forschungshypothesen ${ }^{\prime 20}$ zu gewinnen trachten. Sie lassen sich dadurch charakterisieren, daß sie anders als die Sozialisationstheorien, die im Vorhof der Anthropologie bleiben, über diese hinausgehen und einen gewissermaßen universellen Anspruch erheben. Zu nennen sind etwa das Modell der Homöostase, das Konsistenzmodell, die Systemtheorie, die Kybernetik. Sie alle lassen sich letzten Endes zurückführen auf ein Denkmodell, das sie unterschiedlich modifizieren bzw. weiterentwickeln, nämlich auf das Gleichgewichtsmodell.

Der Begriff der Homöostase, seiner Herkunft nach ein biologischer Begriff, bezeichnet den durch physiologische Kreisprozesse erzielten Gleichgewichtszustand der Organismen, der zur Erhaltung ihres Daseins erforderlich ist. Das gilt für die Phylogenese wie für die Ontogenese der Organismen, es gilt für die einzelnen 
Lebewesen wie für die biologische Lebensgemeinschaft, also für das Zusammenleben von Lebensformen in einer bestimmten abgegrenzten Umwelt, bei der sich im Wettbewerb aller Glieder ein biologisches Gleichgewicht herausbildet und erhält. Dieses Gleichgewicht ist kein statisches, sondern ein dynamisches, d.h. es wird durch Selbstregelung immer neu hergestellt.

Der Gedanke der Kreisprozesse und der Regelung ist auch für die Kybernetik grundlegend; zugleich weitet sie ihn zu einem „gemeinsamen Prinzip“ von Biologie und Technik aus und versucht überdies, ihn als universelles Prinzip und somit als „Brücke zwischen den Wissenschaften“ zu verstehen ${ }^{21}$. So wird etwa auch der didaktische Proze $B$ als Regelungsvorgang interpretiert. Das Wort Kybernetik bezeichnet ursprünglich die Kunst des Lotsen, ein Schiff trotz Wind und Strömung an sein Ziel zu bringen. Dieses Modell des Lotsen, der durch ständig korrigierte Steuerung das vorgegebene Ziel, den Soll-Wert, zu erreichen trachtet und dazu auch ständig Informationen über den Ist-Wert verarbeiten muß, wird zur Erklärung aller Prozesse verwendet, in denen Sicherung oder Regelung notwendig ist. Das sind alle Prozesse der Zielerreichung in offenen, d.h. Störungen unterliegenden Systemen. Charakteristisch für die kybernetische Technik ist die „Automation der Lotsenfunktion“, also die Selbstregelung. Sie läßt sich auch als Regelkreis darstellen.

Die gegenwärtig attraktivste Gestalt des Gleichgewichtsmodells stellt die Systemtheorie dar. Auch sie erhebt einen universellen Anspruch. Als Allgemeine Systemtheorie ${ }^{22}$ beansprucht sie nicht nur für Mathematik und Technik, sondern auch für Biologie, Psychologie, Soziologie etc. zu gelten. Überdies sieht man ihren besonderen Vorzug darin, daß sie nicht nur die Beziehungen eines Systems zu seiner Umwelt zu fassen vermag, sondern auch jene zwischen Gesamtsystem und Teilsystemen. Diese können wiederum in immer kleinere Teilsysteme unterschieden werden bis hin zum psychischen System des individuellen Schülers in einer konkreten Lernsituation, wie es etwa die systemtheoretische Didaktik tut ${ }^{23}$.

Der modernen Systemtheorie geht es also vor allem um die äußeren wie inneren Beziehungen von Systemen; dadurch unterscheidet sie sich vom alten, nur klassifikatorischen Systembegriff. Ein System im modernen Sinne nimmt Umweltereignisse auf und verarbeitet sie. Beide Prozesse sind abhängig von systemimmanenten Strukturen, wenngleich diese Abhängigkeit nicht ausschließt, „daß systeminterne Prozesse auch die Änderung von Strukturen des Systems bewirken können (Lernen) ${ }^{\text {(24 }}$. Da die Umwelt eines Systems stets komplexer ist als das System selbst, ist es auf "Reduktion der Komplexität“ angewiesen. Diese Reduktion bedeutet zugleich Selektion. Durch beides entsteht das, was N. Luhmann „Sinn“ nennt. Sinnerfahrung wird also ermöglicht durch Reduktion von Komplexität, oder anders formuliert: „Sinn ist eine Strategie selektiven Verhaltens unter den Bedingungen hoher Komplexität ${ }^{\star 25}$. Die Gesellschaft schließlich erscheint als „dasjenige Sozialsystem, das Welt und Zeit sinnhaft konstituiert und als Horizont für intersubjektive Prozesse selektiver Kommunikation verfügbar hältt ${ }^{426}$. Schon diese knappen Andeutungen zeigen, daß die Systemtheorie auch anthropologisch höchst fruchtbar ist, aber sie ist eben doch keine genuin anthropologische Theorie (wie sie auch keine zureichende Theorie oder auch nur Definition von „Sinn“ gibt).

Am stärksten in der Nähe einer anthropologischen Theorie angesiedelt sind die sogenannten Konsistenzmodelle, deren bekanntestes L. Festingers Theorie der 
kognitiven Dissonanz ist. Sie wird in der Regel als „Attitüden-Theorie innerhalb der Sozialpsychologie rubriziert ${ }^{* 27}$, tatsächlich ist sie jedoch zunächst den kognitiven Lerntheorien zuzurechnen, die als solche eine Gegenposition zum Behaviorismus darstellen. Insofern bedeutet die Betonung des Kognitiven nicht, daß diese Theorie nur für das Lernen von Menschen gelte, Festinger selbst hat auch das Lernen von „infrahumanen Organismen“ untersucht ${ }^{28}$; umgekehrt bedeutet diese Betonung aber auch nicht, daß menschliches Lernen auf seine kognitive Dimension reduziert würde. Mit Kognitionen sind nicht nur Kenntnisse gemeint, sondern auch Meinungen, Überzeugungen, Wertvorstellungen, Einstellungen, Motive, da sie alle zumindest kognitive Elemente enthalten.

In der Bundesrepublik sind die Konsistenzmodelle einer größeren Öffentlichkeit vor allem im Kontext der Wirkungsforschung bekannt geworden. Hackforth zum Beispiel betrachtet sie geradezu als deren zentrales ,wirkungsrelevantes Ordnungsschema" ${ }^{29}$. Dabei ist Festingers Theorie, die hier stellvertretend für alle Konsistenzmodelle stehen soll, jedoch ungebührlich vereinfacht worden. So schreibt etwa Hackforth: „Im Unterschied zu den lerntheoretischen Ansätzen, bei denen eine Vielzahl motivationeller Zustände Meinungsänderungen auslösen können, wird bei den Konsistenztheoretikern nur von einem Motiv als Bedingung für eine Meinungsänderung durch einen Kommunikationsstimulus ausgegangen, nämlich dem Ungleichgewicht (Inkonsistenz, Dissonanz). “30 Nun gibt es in Festingers Buch gewiß Stellen, die diese Behauptung zu stützen scheinen, und tatsächlich ist inzwischen versucht worden, aus seinem Ansatz eine allgemeine Motivationstheorie zu entwickeln ${ }^{31}$, trotzdem trifft sie nicht zu. Festinger betont vielmehr ausdrücklich: „Es gibt viele Faktoren, die das Verhalten, die Attitüden und die Meinungen der Menschen beeinflussen, über die die Theorie der Dissonanz nichts aussagt. So haben wir z.B. in diesem Buch nichts oder nur wenig über Motivation gesagt. Dissonanz kann natürlich als ein motivierender Faktor angesehen werden, doch gibt es eine Vielzahl weiterer Motive, welche die Menschen beeinflussen."32 Vielleicht haben die Vereinfachungen auch mit der Tatsache zu tun, daß sein bereits 1957 erschienenes Hauptwerk "A Theory of Cognitive Dissonance" erst 1978 in deutscher Übersetzung erschienen ist.

Aus dem Gesagten ergibt sich bereits, daß Festingers Theorie mit wenigen Sätzen nicht ange messen darzustellen ist. Doch sollen wenigstens seine Grundthesen skizziert werden. Er geht gleich im ersten Satz von der Feststellung aus, „daß der Mensch nach Konsistenz strebt" 33 , übernimmt jedoch nicht die Begriffe Konsistenz oder Gleichgewicht als grundlegend, sondern entscheidet sich für den der Konsonanz, weil dieser ihm weniger belastet erscheint. Die Begriffe Konsonanz und Dissonanz weisen auf Beziehungen hin, die zwischen Paaren von kognitiven „Elementen" bestehen; diese Elemente wiederum beziehen sich „, auf Dinge, die eine Person über sich selbst, über ihr Verhalten und über ihre Umwelt wei $\beta^{\text {“34 }}$; zugleich reagieren die Elemente „auf die Realität“. Wenn sie nun in einer dissonanten Beziehung zueinander oder zur Realität stehen, erzeugt diese Dissonanz „Druck zur Reduktion oder Beseitigung der Dissonanz. Die Stärke des Drucks zur Dissonanzreduktion ist eine Funktion der Stärke der Dissonanz“, diese wiederum „eine Funktion der Wichtigkeit der Elemente“. Weil die Dissonanzstärke entscheidend ist, kann zugleich gelten, was in Sekundärdarstellungen häufig ignoriert wird, „daß eine gewisse Dissonanz für gewöhnlich die Regel ist“"35. 
Es gibt „drei wesentliche Wege“ der Dissonanzreduktion: 1. Die Änderung eines oder mehrerer Elemente, die an den dissonanten Beziehungen beteiligt sind. 2. Das Hinzufügen neuer kognitiver Elemente, die mit bereits bestehenden Kognitionen konsonant sind. 3. Das Verringern der Wichtigkeit der an den dissonanten Beziehungen beteiligten Elemente ${ }^{36}$. Diese drei wichtigsten Möglichkeiten werden von Festinger in 12 Unterpunkten konkretisiert. Darin taucht auch die „Rolle der sozialen Unterstützung" auf, der er immerhin zwei der elf Kapitel seines Buches gewidmet hat: Dissonanz läßt sich auch dadurch reduzieren, daß man Kontakt mit Personen sucht, die „die gleiche Dissonanz erleiden“. Schließlich muß noch erwähnt werden, daß Festinger ausdrücklich auch auf Persönlichkeitsunterschiede hinweist ${ }^{37}$. "Für manche Menschen ist Dissonanz eine extrem schmerzhafte und unerträgliche Angelegenheit, während es andere gibt, die anscheinend imstande sind, eine sehr starke Dissonanz zu ertragen." Entsprechend unterscheidet er zwischen Personen mit hoher und solchen mit niedriger „Dissonanztoleranz“.

Die Bedeutung der Festingerschen Theorie für die Fernsehwirkungsforschung wird in der Sekundärliteratur etwa so charakterisiert: „Der Mensch versucht, in seinem Wahrnehmen und Verhalten Dissonanzen zu vermeiden zwischen dem, was ihm in der Umwelt begegnet, und der präexistenten Attitüdenstruktur ... Dieser Vorgang hat weitreichende Konsequenzen für die Wirkungen des Fernsehens. Viele Sendungen erreichen überhaupt nicht das angezielte Publikum, weil die potentiellen Rezipienten schon vorher annehmen und befürchten, durch diese Sendungen in ihren bereits vorhandenen Meinungen und Attitüden verwirrt zu werden; sie sehen sich solche Sendungen erst gar nicht an." Aber nicht nur in der Auswahl dessen, was man auf sich einwirken lassen will, auch im Wahrnehmen, Erleben, Verarbeiten und Bewerten der Aussage schlägt sich das Streben nach kognitiver Konsonanz nieder. „Die Beeinflussung ist in der Regel um so stärker, je mehr die empfangenen Aussagen mit den schon vorhandenen Attitüden beim Zuschauer übereinstimmen. Starke Wirkungen sind auch möglich, wenn sich der Zuschauer auf dem Gebiet, von dem eine Sendung handelt, noch nicht festgelegt hat. Bei einer Dissonanz und den präexistenten Dispositionen kommen oft gar keine Wirkungen zustande, oder die Wirkungen laufen sogar den Intentionen des Kommunikators zuwider (Bumerang-Effekt). ${ }^{\text {"38 }}$ Kognitive Dissonanzen lassen sich aber auch dadurch vermeiden, daß dissonante Informationen übersehen, für unwahr gehalten, schnell wieder vergessen, in konsonante umgedeutet oder so minimalisiert werden, daß sie ihre Bedeutung verlieren.

Auf die Kritik an Festingers Theorie ist hier nicht mehr einzugehen ${ }^{39}$. Wenigstens erwähnt werden soll jedoch, daß auch J. Piaget, dessen Theorie besonders durch $\mathrm{H}$. Sturm für die Medienforschung und Medienpraxis fruchtbar zu machen versucht wird $^{40}$, den kognitiven Lerntheoretikern zugezählt wird, daß Piaget, seiner Ausbildung nach Biologe, vor allem aber ein geradezu klassischer Vertreter des alten Homöostasemodells ist, dem er freilich eine spezifische Gestalt gegeben hat. Das Gleichgewicht zwischen dem Organismus und seiner Umwelt wird durch wechselseitige Anpassung erreicht. Zum einen werden die Umweltreize den vorhandenen kognitiven Strukturen einverleibt; Piaget bezeichnet diesen Vorgang als Assimilation. Zum anderen werden die vorhandenen Strukturen unter dem Druck der Umwelt erweitert und differenziert, und zwar so, daß sie der Realität besser gerecht werden als vorher; diesen Vorgang nennt Piaget Akkommodation. Auf diese Weise geschieht der eigentätige Aufbau stets neuer Strukturen, ein stufenförmiger 
Aufbau, dessen Stufen wiederum dadurch gekennzeichnet sind, „daß ein relatives Ungleichgewicht an ihrem Anfang und der Vorgang des Aufbaus und der Festigung eines bestmöglichen Gleichgewichts in ihrem Mittelpunkt steht; am Ende dann wird dieses wieder überwunden, und ein neues Gleichgewicht, nun auf einem höheren Strukturniveau, wird aufgebaut" ${ }^{\prime 4}$. Nach den „optimalen Gleichgewichtsformen" werden dann die großen Entwicklungsstufen des Kindes benannt als Stufe der sensomotorischen Intelligenz, der konkreten Operationen, der formalen Operationen. Schließlich taucht das Gleichgewichtsprinzip noch einmal unter dem Begriff „Äquilibration“ auf. Damit ist der „innere Mechanismus“ gemeint, der den Entwicklungsproze $ß$ steuert. Die drei anderen beteiligten Faktoren (organisches Wachsen und Reifen, handelnder Umgang mit den Gegenständen, soziale Interaktionen und erzieherische Übermittlungen) bedürfen der Lenkung, damit die Invarianz der Stufenabfolge zustandekommt. Dieses leistet die Äquilibration, „ein Ausgleichsprozeß, nicht im Sinne eines bloßen Gleichgewichts der Kräfte wie in der Mechanik ..., sondern im Sinne, wie es heute dank der Kybernetik bekannt ist, einer Selbstregulierung “42.

Selbstverständlich bedürfen die skizzierten Theorien einer gründlicheren Darstellung und Würdigung. Im Rahmen unserer Fragestellung sollte jedoch nur zu zeigen versucht werden, daß die verschiedenen Konkretisierungen des Gleichgewichtsmodells zwar anthropologisch höchst fruchtbar, aber eben keine anthropologischen Theorien sind, deshalb auch nicht den gesuchten integrativen Rahmen abgeben können. Könnte es nicht sein, daß man spezifisch Menschliches gerade nicht $\mathrm{zu}$ fassen bekommt, wenn man den Menschen und sein Verhalten mit Hilfe universeller Modelle zu erklären versucht? Warum strebt der Mensch nach Konsonanz? Ist dieses Streben so grundlegend, daß man von ihm her sein gesamtes Verhalten erklären kann? Ist die Selbstregulierung des Menschen von gleicher Art wie die von Thermostaten, Pflanzen, Tieren? Kann es nicht gerade für die Massenkommunikationsforschung von vornherein von Übel sein, wenn man den multifunktionalen Charakter menschlichen Kommunikationsverhaltens, also auch der Mediennutzung, ignoriert?

\section{Umrisse einer anthropologischen Rabmentheorie}

Auf den ersten Blick mag es scheinen, als sei gerade die anthropologische Fragestellung völlig ungeeignet, hier weiterzuhelfen. Gibt es Kontroverseres als die Antworten auf die Frage nach dem Menschen? Sie hängen ab zum einen von der Perspektive der Einzelwissenschaften, die sich mit dem Menschen beschäftigen, zum anderen vom jeweiligen eigenen philosophischen oder ideologischen Standort. Die alte philosophische Überzeugung von der menschlichen Natur als fester Größe, als übergeschichtlicher, allgemein gültiger Form und Norm, gehört jedenfalls spätestens seit Dilthey der Vergangenheit an. Zwar bekennen wir uns auch heute noch zu übergeschichtlichen Strukturen des Menschlichen, zum Beispiel in Gestalt der „Naturrechte“ des Menschen, aber der aktuelle Streit um deren geschichtliche, politische, gesellschaftliche, kulturelle Auslegung ist aufschlußreich genug. „Um das Übergeschichtliche zu erkennen, muß der Mensch die Geschichte des Übergeschichtlichen kennen", hat K. Pawek einmal treffend formuliert ${ }^{43}$.

Doch trotz vieler Kontroversen gibt es durchaus auch Konsens. So wird von niemandem bestritten, daß der Mensch das einzige Wesen ist, das nach sich selbst fragen, 
das überhaupt Fragen stellen kann ${ }^{44}$. Schon Nietzsche meinte, der Mensch sei das nicht festgestellte Tier, und damit ist wohl auch gemeint, er sei das nicht feststellbare, sondern stets „hinterfragbare“ Wesen. Heute bezeichnen die einen diesen Sachverhalt als die Plastizität der Natur des Menschen, während stärker philososphisch orientierte Autoren formulieren, er bleibe trotz des wachsenden empirischen Wissens eine „offene Frage“, die durch keinen Begriff, keine Theorie, kein Menschenbild jemals erschöpfend zu beantworten ist. Die „Fraglichkeit" ist also offensichtlich für den Menschen konstitutiv, folglich stellt sie ein spezifisch anthropologisches Prinzip dar. Zugleich ist sie ein grundlegendes Prinzip, da sie die einzige Voraussetzung bildet, hinter die wir in unserem Fragen nicht mehr zurückgehen können ${ }^{45}$.

Diese „Fraglichkeit“ muß freilich sogleich konkretisiert werden, und zwar in zweifacher Richtung. Einmal wird dadurch ausgedrückt, daß der Mensch das in Frage gestellte, das fragwürdige Wesen im negativen (metaphorischen) Sinn dieses Begriffs ist, das mängelhafte und gefährdete, weil nicht umweltgebundene und instinktgeleitete Wesen. Zum anderen wird der Mensch gefaßt als in die Möglichkeit der Frage hineingestellt, als das fragwürdige Wesen im positiven (wörtlichen) Sinn des Wortes, also als das des Fragens würdige, durch die Möglichkeit des Fragens ausgezeichnete Wesen. Entscheidend ist dabei, daß beide Auslegungen nur zwei Aspekte der einen Grundstruktur des Menschen darstellen und deshalb untrennbar sind, daß also die naturhafte Not des „In-Frage-gestellt-seins“ und das „In-die-Möglichkeit-der-Frage-hineingestellt-sein" aus einer Wurzel entspringen.

Die These vom Menschen als Mängelwesen ist in der Gegenwart von A. Gehlen ausgearbeitet worden. Die Grundlinien seiner Argumentation sind jedoch uralt, sie finden sich bereits in der griechischen Philosophie. Am ausführlichsten sind sie dargestellt in Platons Dialog Protagoras, dort allerdings in die Form des Mythos gekleidet. Als die Götter die sterblichen Wesen schufen, so trägt der Sophist Protagoras vor, beauftragten sie den Epimetheus, er solle sie mit verschiedenen Fähigkeiten ausstatten. Epimetheus gibt den einen Stärke, den anderen Schnelligkeit, er verteilt ausgleichend die verschiedenen Mittel zum Schutz gegen die Witterung, weist ihnen verschiedene Nahrung zu, bedacht, jede Art vor Ausrottung zu schützen. Doch plötzlich ist er mit seinen Gaben zu Ende, ,und nun blieb ihm noch unausgestattet das Menschengeschlecht übrig, und er war ratlos, was er diesem tun sollte“. Da kommt ihm Prometheus zu Hilfe, er stiehlt den Göttern das Feuer und schenkt es dem Menschen, damit er technisch handelnd seine Unfähigkeit zur biologischen Daseinserhaltung kompensieren kann. Aber das Geschenk der Technik genügt noch nicht, da sie zur Regelung es menschlichen Zusammenlebens nicht taugt, und so droht ihm immer noch der Untergang. Deshalb schenkt Zeus den Menschen noch die „bürgerlichen Tugenden“ (also das, was wir heute als Institutionen bezeichnen), die sie zu Gemeinschaftswesen machen, „damit es in den Städten feste Ordnungen gebe“.

Diese Argumentation, die auch den Kirchenvätern (Gregor v. Nyssa) und der Scholastik (Thomas v. Aquin) bekannt ist, und die sich in der Neuzeit vor allem bei Herderfindet ${ }^{46}$, wird von Gehlen aufgegriffen. Auch er geht von der organischen Mängelhaftigkeit des Menschen aus und stellt fest, ,der Mensch wäre, der rohen Natur wie ein Tier ausgesetzt, mit seiner ihm angeborenen Physis und seiner Instinktmangelhaftigkeit unter allen Umständen lebensunfähig “47. Er fragt dann weiter, mit welchen Mitteln ein derartig ausgestattetes Wesen eigentlich existiert. Da er sich 
auf diesen Frageansatz beschränkt, kann er den Menschen jedoch nur noch im Horizont seiner biologischen Natur sehen, deren funktioneller Sinn sich darin erschöpft, das von ihr produzierte Leben vor dem natürlichen Untergang zu bewahren.

Dennoch führt dieser reduzierte Ansatz, der das Produktive der menschlichen Daseinsweise nur als Kompensation des natürlichen Mangels deutet, zu einigen wichtigen Einsichten. Als weltoffenes Wesen von außen der Reizüberflutung, von innen dem Antriebsüberschuß ausgesetzt, braucht der Mensch die Fähigkeit zu distanzierender Entlastung, um leben zu können. Und Gehlens origineller Einfall ist es nun, die Gesamtausstattung des Menschen von der Sensomotorik über die Sprache bis zum Denken als ein Gefüge von Entlastungsstrukturen durchzukonstruieren. Technik und Kunst, die Gesamtheit der Institutionen werden ebenso wie die individuell erworbene Wahrnehmungswelt unter dem Aspekt der Entlastung begriffen.

Gehlen hat in überzeugenden Analysen gezeigt, wie dieser eigentätige Aufbau der Wahrnehmungs- und Erfahrungswelt vor sich geht, wie insbesondere die menschliche Sensomotorik für diese Aufgabe gerüstet ist, wie mit der Durcharbeitung und Aneignung der Welt in ihrer Fülle zugleich eine zweite Aufgabe gelöst wird, nämlich sich selbst anzueignen, „eine Reihe beherrschter Leistungen“auszubilden, der eigenen Plastizität Gestalt zu geben ${ }^{48}$. So ist die Wahrnehmungswelt, der scheinbar „unmittelbare“ Bestand der gegebenen Welt also in Wahrheit „hochgradig“ durch unsere Eigentätigkeit vermittelt und geradezu ein Resultat ${ }^{49}$. Nachdem wir im handelnden Umgang mit den Dingen deren Beschaffenheit begriffen haben, brauchen wir nicht jedesmal neu nach ihnen zu greifen, vielmehr werden ihre Umgangs- und Gebrauchswerte dann vom Gesichtssinn übernommen, ihre Materialbeschaffenheit, Schwere, Entfernung, ja ihre „Handlichkeit“, die wir überdies auch noch sprachlich „fassen“, „festhalten“, auf den „Begriff“ bringen können - selbst die Alltagssprache macht den Zusammenhang dieser Tätigkeit mit der Sensomotorik deutlich. Am Ende dieser hier nur angedeuteten Prozesse steht als Ergebnis, daß wir uns die Welt übersichtlich gemacht haben und uns deshalb in ihr orientieren können.

Aber auch die Normen, Konventionen, Verhaltensmuster jener Kultur und Gesellschaft, in die der Mensch hineingeboren wird, müssen vom einzelnen selbsttätig erworben werden. Nur auf diese Weise kann er, da instinkthaft nicht festgelegt, die Sicherheit des gesellschaftlichen Verhaltens gewinnen. Die kulturellen Verhaltensmuster oder Institutionen, die erst ein dauerhaftes Zusammenleben von Menschen ermöglichen, haben also für den einzelnen eine stabilisierende Funktion: „Sie sind die Formen, die ein seiner Natur nach riskiertes und unstabiles, affektüberlastetes Wesen findet, um sich gegenseitig und um sich selbst zu ertragen, etwas, worauf man in sich und den anderen zählen und sich verlassen kann." Nicht zuletzt bringen sie den „außerordentlichen Gewinn einer Stabilisierung auch des Innenlebens “60, so daß man sich nicht ständig neu affektiv auseinanderzusetzen oder sich Grundsatzentscheidungen abzuzwingen hat. Auch die kulturellen und gesellschaftlichen Verhaltensmuster dienen folglich insofern der Orientierung des einzelnen, als sie Welt und Gesellschaft übersichtlich machen und ihn vom Zwang permanenter Neuorientierung befreien. Er erreicht auf diese Weise eine „wohltätige Sicherheit“, die lebenswichtig für ihn ist.

Manche Kritiker behaupten, bei Gehlen gerate die Genese des Menschen notgedrungen zu einem Prozeß totaler Anpassung, da die verlorene Instinktsteuerung 
vollständig durch sozialisierte Zwänge ersetzt werde ${ }^{51}$; andere sehen in dieser Kritik ein Mißverständnis von Gehlens Institutionenlehre, „vielleicht nicht ohne seine Schuld“52. Auf diese Kontroverse ist hier nicht einzugehen; er selbst sagt, daß erst „aus diesem Unterbau innerer und äußerer Gewohnheiten die geistigen Energien sozusagen nach oben abgegeben werden können“, für „eigentlich persönliche, einmalige und neu zu erfindende Dispositionen" frei werden ${ }^{53}$. Die Kontroverse hat jedenfalls damit zu tun, daß er nur den negativen Aspekt menschlicher Fragwürdigkeit thematisiert, der sich ergibt, wenn man den Menschen von der biologischen Naturordnung her betrachtet.

Der positive Sinn der Fragwürdigkeit hingegen wird deutlich, wenn man das Produktive der menschlichen Daseinsweise nicht nur als Kompensation seiner biologischen Ungesichertheit versteht, sondern auch als Ausdruck seiner geistigen Freiheit. Sie besteht darin, daß der Mensch trotz aller Abhängigkeiten nicht schlechthin eingebunden ist in Natur, Kultur, Gesellschaft; daß er von diesen wie auch von den erworbenen Verhaltens- und Orientierungsweisen nicht schlechthin bestimmt wird. Er kann vielmehr grund sätzlich alles in Frage stellen, auch dasjenige, was ihm so selbstverständlich geworden ist, daß es die Überzeugungskraft des Natürlichen gewonnen hat - insofern ist es aufschlußreich, daß wir in der Alltagssprache ständig die Bezeichnung „natürlich“ gebrauchen, wenn wir ,selbstverständlich“ meinen. Dabei denkt der Mensch sich die Fragen nicht aus, aus welchen Gründen auch immer; indem er sie stellt, werden sie ihm zugleich gestellt, nämlich von der Wirklichkeit, in der er lebt. Dem Problembewußtsein, aus dem die Fragen kommen, korrespondiert, daß dem Fragenden etwas problematisch geworden ist.

Ein bekanntes Argument der Reformpädagogik gegen die Schule lautet, dort frage ständig der Lehrer die Schüler, also ein Wissender die Unwissenden; vernünftigerweise aber müsse es umgekehrt sein. So einleuchtend das Argument auf den ersten Blick scheint, tatsächlich trifft es nur die halbe Wahrheit. Denn um fragen zu können, braucht man ein Problembewußtsein; genau das aber soll ja von der Schule, nicht zuletzt durch die „richtige“ Lehrerfrage, erst entwickelt werden. Gewiß muß jeder seine Fragen selber stellen, aber initiiert wird die Fragebewegung von außen, sei es durch die Fragwürdigkeit der Gegebenheiten selbst oder durch einen anderen, der auf sie aufmerksam macht.

„Fragen sind so wichtig im menschlichen Leben, daß sie sogar in der Sprache eine Sonderform haben. " 54 Nur durch Fragen und Weiterfragen kann der Mensch die für ihn wesentliche Dimension fortschreitenden individuellen Sinngewinns realisieren, und auch gesellschaftliche und kulturelle Weiterentwicklung ist nur auf diese Weise möglich. Die von Gehlen erwähnten geistigen Energien, die „sozusagen nach oben abgegeben werden können", werden erst durch Fragen mobilisiert. Nun könnte man gegen das bisher Gesagte anführen, es sei im Grund nur eine Variation des biologischen oder systemtheoretischen Gleichgewichtsmodells, die Frage erscheine hier letzten Endes als eine Art Gleichgewichtsregulator. Nun sollen hier Gemeinsamkeiten gar nicht bestritten werden, es gilt jedoch den Unterschied zu sehen. Das Gleichgewichtsmodell ist ein universelles - und eben daraus ergeben sich die Gemeinsamkeiten -, die Frage hingegen ist ein spezifisch anthropologisches Prinzip, das deshalb auch im Hinblick auf den Fernsehzuschauer präziser und ergiebiger sein müßte. 
Doch bevor wir das bisher Entwickelte auf den Fernsehzuschauer zu übertragen versuchen, ist noch einmal zu betonen, daß die beiden skizzierten Aspekte nicht etwa als Gegensatz aufzufassen sind, sondern als Einheit. Allerdings bilden sie eine ausgesprochen spannungsvolle Einheit. Einerseits kann der Mensch nicht ständig alles in Frage stellen und von Grund auf neu begreifen und begründen, denn das würde ihm zu leben unmöglich machen; andererseits macht es gerade seinen Vorzug gegenüber allen anderen Lebenwesen aus, daß es des Fragens würdig ist. Einerseits ist die wohltätige Sicherheit, die ihm seine erworbenen Verhaltensmuster gegenüber Welt und Mitmenschen geben, für ihn lebenswichtig; andererseits ist nur er in der Lage, auch zu diesen seine Lebensführung bestimmenden Verhaltensmustern (sogar zu seiner eigenen Lebensgeschichte) in kritische Distanz zu treten, sie in Frage zu stellen, sie dadurch veränderbar zu machen und sie auch tatsächlich eigentätig und selbstverantwortlich zu verändern, also nicht nur zu „funktionieren“.

Beide Sichtweisen bilden eine spannungsvolle Einheit, und dies macht verständlich, daß sie in der Geschichte abendländischen Denkens als „naturalistischer“ und „idealistischer“ Ansatz einander feindlich gegenüberstehen. Entweder geht man von der Not der In-Frage-gestelltheit der biologischen Natur des Menschen aus; dann erscheint die unerhörte Möglichkeit seines Fragenkönnens nur als Kompensation seiner biologischen Mängelhaftigkeit, als Möglichkeit zum Überleben. Oder man geht umgekehrt von seinem Fragenkönnen als der Chance seiner geistigen Freiheit aus; dann scheint sich die Not seiner physischen Existenz bis zur Bedeutungslosigkeit zu verflüchtigen. „Natur" und „Geist" im Menschen sind jedoch nicht als Gegenspieler zu fassen, auch nicht als übereinander geschichtete Realitäten, sondern als ursprüngliche, unauflöslich miteinander verzahnte Einheit. Der Sinngewinn durch vermittelndes Fragen ist nicht auf die Reflexion des Menschen eingeschränkt, er ist bereits in seiner Leiblichkeit grundgelegt, wie sich insbesondere am Beispiel der Sensomotorik zeigt; die biologischen Gegebenheiten setzen dem fragenden Subjekt nicht nur natürliche Grenzen, sondern gehen auch als Antriebe in sein Fragen ein. So kommt der Gedanke der Freiheit und Selbstbestimmung ebenso zu seinem Recht wie die Bedürftigkeit der menschlichen Leibes- und Gattungsnatur. „Die Not eines Infragegestelltseins wird auf dem Weg eines sinnaufschließenden Erfragens behoben und als neuer Horizont menschlicher Einsicht und Haltung eingebracht." 55

\section{Allgemeine Konsequenzen für den Fernsehzuschauer}

Überträgt man die bisherige Argumentation auf den Menschen als Fernsehzuschauer, so ergeben sich mehrere Anknüpfungspunkte. So kann man einsetzen bei der Tatsache, daß das Fernsehen eine Rolle spielt bei der Gestaltung seines Alltags, wie die verschiedenen Tagesablaufuntersuchungen belegen. Es formt seine Lebensgewohnheiten mit, und es kann dabei sogar zu einem dominierenden Faktor werden, so daß sein Ausfall zu einer Art von Entzugserscheinungen führt, wie das mit zwei Berliner Arbeiterfamilien durchgeführte Experiment ,Vier Wochen ohne Fernsehen" gezeigt hat. Nun ist aber daraus nicht vorschnell ein Argument gegen das Fernsehen abzuleiten, denn ähnliche Phänomene können auftreten, wenn andere äußere Stützen oder Entlastungsstrukturen wegfallen, die Tageszeitung, das liebste Hobby, der Freundeskreis, wenngleich freilich das Fernsehen allein schon wegen seines festen Programmschemas ein ungleich stärker den Alltag strukturierender Faktor ist. Dennoch vermissen auch abhängig gewordene Zuschauer den 
Bildschirm in der Regel nicht, wenn sie in den Urlaub fahren, denn der Reiz des Urlaubs besteht ja gerade darin, daß man einmal anders lebt als im häuslichen Alltag. Daran zeigt sich, daß offenbar weniger der Fernsehentzug das Problem ist als vielmehr die damit verbundene abrupte Umstellung der alltägllichen Lebensgewohnheiten.

Andere Aspekte sind zentraler. Der Mensch muß sich seine sämtlichen Orientierungs- und Verhaltensweisen selbst aufbauen und auf diese Weise die Welt übersichtlich machen. In der konkreten Situation verläßt er sich auf diesen erworbenen Bestand, und es ist für ihn lebenswichtig, daß er sich darauf auch verlassen kann. Mit anderen Worten: Übersicht kommt erst durch das Übersehen zahlloser auch möglicher Wahrnehmungen zustande; Gehlen hat auf diesen "tiefen Doppelsinn“ des Wortes Übersicht hingewiesen ${ }^{56}$. Die Rezeption des wahrnehmenden Subjekts besteht demnach vor allem im Vereinfachen, in der Reduktion der Eindrucksfülle und ihrer Integration in das Raster der vorhandenen Orientierungsweisen. Es besteht offenbar sogar die Neigung, bis zur bloßen Zweiteilung zu vereinfachen, Übersicht also durch bloße Dichotomien zu erreichen. Jedenfalls ist auffällig, mit welcher Selbstverständlichkeit und Hartnäckigkeit wir uns solcher simplen Zweiteilung bedienen - selbst die sogenannten Intellektuellen.

Für ein Wesen mit so hoher Reizzugänglichkeit, wie der Mensch es ist, für ein weltoffenes Wesen also, ist ein solches grobes Einteilungsraster offenbar wichtig. Je komplexer und undurchschaubarer, je fremder und beunruhigender eine Situation ist, mit der er konfrontiert wird, um so nachdrücklicher bedient er sich des Schemas der bloßen Zweiteilung, um sich eine erste Grundorientierung und damit Sicherheit zu verschaffen. Das gilt gegenüber der Komplexität der realen Umwelt, aber auch gegenüber der Welt, die das Fernsehen ihm vermittelt. Es sind die bekannten Einteilungen wie richtig - falsch, wahr - unwahr, progressiv - reaktionär, kapitalistisch - proletarisch, oder wie immer dieses Grobmodell jeweils inhaltlich gefüllt wird. Auch die Begriffe Gleichgewicht - Ungleichgewicht könnten hier übrigens genannt werden. Dabei ist auffällig, daß es bei diesen Einteilungen im Grund um Wertungen geht, die Zustimmung oder Ablehnung bedeuten. Wir operieren also offenbar besonders dann mit ihnen, wenn wir uns existentiell angesprochen fühlen, in unseren Grundeinstellungen betroffen werden, im Unterschied zu solchen Situationen und Informationen, die für unser Welt- und Selbstverständnis nur peripher sind. Auf diesen wichtigen Tatbestand ist gleich noch eigens einzugehen.

Zuvor ist jedoch noch auf andere, weniger grobschlächtige Orientierungsarten hinzuweisen. Zu nennen sind insbesondere die Stereotype und Klischees. Sie können unterschiedlich fixiert, aber auch unterschiedlich plump bzw. sogar relativ differenziert sein. Auch sie haben eine für den Menschen bedeutsame entlastende Funktion und sind deshalb unausrottbar. Denn auch sie dienen gewissermaßen als Stützen der Welt- und Verhaltensorientierung, indem sie komplexe Sachverhalte und Geschehnisse auf einige einfache Grundmuster reduzieren. Die Neigung, sich an Stereotype zu halten, weist also wiederum auf Grundbedürfnisse eines weltoffenen Wesens hin. Der Mensch kann, zugespitzt gesagt, ohne solche Stereotype nicht leben; dies freilich macht nur die eine Seite seiner Weltoffenheit aus, deren andere darin besteht, daß er wie alles so auch sie in Frage stellen und sie dadurch aufbrechen kann, daß er auf diese Weise wieder eine offene Beziehung zu Dingen, Menschen und Ereignissen gewinnen kann. Allerdings bedeutet das keine Überwin- 
dung von Stereotypen schlechthin; an die Stelle der alten treten neue, differenziertere und adäquatere, aber eben doch wieder sterotype, weil nur so entlastende Muster. Gerade in einer immer komplizierter werdenden Welt ist anderes gar nicht möglich.

Kein Zweifel, daß Stereotype weitgehend gesellschaftlich bzw. gruppenspezifisch bedingt und tradiert sind. Man kann sie aber auch ausdrücklich unter dem Gesichtspunkt ihrer gesellschaftlichen Sanktionierung betrachten. Dann zeigt sich, daß die das menschliche Zusammenleben regelnden Institutionen auch nichts anderes sind als Sterotype. Man muß ja bei diesen Institutionen nicht sogleich an Recht und Moral denken, so zentral sie sind, man kann auch an so etwas Banales wie die Verkehrsregeln denken, von denen gleichwohl das Leben der Verkehrsteilnehmer einschließlich der Fußgänger abhängt.

Obwohl gesellschaftlich bedingt, sind die Stereotype doch nicht ausschließlich gesellschaftlicher Natur. Da sie eigentätig erworben sind, geht auch die Individualität des Subjekts in sie und damit in Wahrnehmen und Verhalten ein. Das gilt im umfassenden Sinne. Wenn etwa ein Laie und ein Experte unter die Motorhaube ihres Autos sehen, so ist ihre Wahrnehmung höchst unterschiedlich, und auch jene der Experten untereinander kann beträchtlich differieren (darauf u.a. beruht die unterschiedliche Qualität von Reparaturwerkstätten); dasselbe gilt für die Wahrnehmungen, die Förster, Jäger, Flüchtlinge, Militärstrategen, Spaziergänger etc. im Walde machen; es gilt auch für die Wahrnehmung gesellschaftlicher Gegebenheiten. Die in der individuellen Genese erworbenen Orientierungs- und Verhaltensweisen, Einstellungen und Überzeugungen bilden das Raster, in das Informationen und Ereignisse, unmittelbar erfahrene wie durch Medien vermittelte, integriert werden oder durch das sie hindurchrutschen, sei es im Sinne der Selektivität der Wahrnehmung, sei es in der Weise, daß sie schnell wieder vergessen werden. Es ist in der ursprünglichen Plastizität und Nichtfestgestelltheit des Neugeborenen begründet, daß der Mensch, als Gattung wie als Individuum, höchst unterschiedliche Verhaltensstrukturen aufbauen kann. Sie alle aber funktionieren in der gleichen Weise, und vor allem sind sie unentbehrlich.

Das Gesagte gilt auch für die Sprache des Menschen. Zum einen ist sie das Werk der Gattung Mensch, das Ergebnis ihrer Auseinandersetzung mit der Wirklichkeit, ist geistige Gestaltung der Welt. Insofern dient auch sie der entlastenden Orientierung, zugleich ermöglicht sie die Kommunikation über diese Welt. Zum anderen hat sie für das Individuum eine welterschließende Kraft, ist also nicht nur „ergon”, sondern auch „energeia”, wie schon W. v. Humboldt formulierte. Sie ist das freilich nur in dem Maße, in dem das Individuum sich in die ihm vorgegebene Sprache der Sprachgemeinschaft, in die es hineingeboren wird, einarbeitet, sie eigentätig erwirbt, sich die in ihr gespeicherte Erfahrung aneignet bzw. die eigene Erfahrung in sie einbringt. Insofern hat auch die Sprache, die derSprachgemeinschaft wie die des Individuums, einen selektiven Charakter - ein Tatbestand, der für die Massenkommunikation überaus wichtig ist, auf den im Rahmen dieser Arbeit jedoch nicht näher eingegangen werden kann ${ }^{57}$.

Kürzlich hat U. Saxer verschiedene Selektionsmodalitäten unterschieden. Er stellt zunächst fest, ihm sei in der Kommunikationswissenschaft und den mit Selektivität sonst befaßten Disziplinen zwar „keine verbindliche Typologie von Selektivitätsrichtungen bekannt", doch könne man „von den dominierenden Befunden der Rezep- 
tionsforschung aus“ vier Selektionsarten unterscheiden. 1. Selbstbestätigende Selektivität, die bestehende Meinungen und Einstellungen stabilisiert. „Die geistige Produktivität dieser Art von Selektivität ... ist zumindest problematisch.“2. Eskapistische Selektivität, die im Sinne einer „Entproblematisierung von Sachverhalten und Streßmilderung “ funktioniert. 3. Sozial gerichtete Selektivität, die Medien nutzt, um daraus Stoff für interpersonale Kommunikation zu gewinnen. 4. Kognitiv erweiternde Selektivität ${ }^{58}$. Saxer faßt hier den Selektionsbegriff zwar weiter, als wir es getan haben, trotzdem ist leicht zu sehen, daß seine Unterscheidung sich nahtlos unserer Argumentation einfügt.

Wie schon angemerkt, ist noch ausd rücklich auf das Problem der Grundeinstellungen einzugehen. Daß Technik und Institutionen für den Menschen lebenswichtig sind, ist ein uralter Gedanke, der wie dargelegt schon von Platon überliefert wird. In unserer Zeit nun hat E. Lemberg ein weiteres „Leit- und Steuerungssystem" unterschieden: die ideologischen Systeme. Er stellt sie ausdrücklich als dritte spezifisch menschliche Errungenschaft neben Technik und Institutionen. Er fragt also nicht nach dem Wahrheitsgehalt der Ideologien, das ist für ihn erst die zweite Frage, sondern nach ihrer anthropologischen Funktion. Unter ideologischen Systemen versteht er „ein Gefüge von Vorstellungen, Werten und Normen, die den Menschen in der Welt orientieren, ihm eine Rolle darin zuweisen, sein Handeln in Gang setzen und sein Verhalten steuern ${ }^{59}$. Es sind geistige Systeme, „die dem Menschen Welterklärung, Verhaltenssteuerung und Sinndeutung gewähren“60. Zu diesen Systemen zählt er auch die Religionen, die allerdings insofern eine Sonderstellung einnehmen, als sie zum einen die Fähigkeit des Menschen zur Transzendenz „voll ausschöpfen“, zum anderen „durch den Ideologiebegriff bei weitem nicht definiert" sind ${ }^{61}$; gewissermaßen am anderen Ende des Spektrums stehen die Weltanschauungen und Parteidoktrinen, verschieden nach Reichweite, Verbindlichkeit und Inhalt.

Nun können seine hochinteressanten Analysen und Argumentationen hier nicht wiedergegeben werden. Nur seine Grundthese ist festzuhalten, daß der Mensch auch ohne Ideologien nicht leben kann, daß sein Grundbedürfnis nach „Glaubenswahrheit" nicht durch die moderne Wissenschaft befriedigt werden kann, da sie ihre Erfolge ja gerade der Tatsache verdankt, daß sie die Sinnfrage suspendiert hat. Wenn aber die Wissenschaft verspricht, die bloße Glaubenswahrheit durch Erkenntniswahrheit zu überwinden, so bleibt entweder ein Vakuum, oder die Wissenschaft wird selbst wiederum zum ideologischen System, wie Lemberg am Beispiel der Aufklärung zeigt. Ganz zu schweigen von jenen vorwiegend deutschen Intellektuellen, nicht selten Gelehrten von Rang, die die Welt ,in eigene Wahrheit und fremde Ideologie“ aufteilen, „die von solcher Gegenüberstellung ihre Autorität ableiten, breite Scharen von Studenten mit dem stolzen Bewußtsein kritischen Denkens erfüllend, ihre eigene Denkmethode in naiver Selbstreklame als kritische Theorie verkündend, sei sie auch weit über ihre Schule hinaus als eine ausgesprochene Heilslehre erkennbar, als ein typischer Fall von Ideologie" ${ }^{\text {662 }}$.

Das Glaubensbedürfnis ist also keineswegs nur für den Menschen charakteristisch, der wissenschaftlicher Erkenntnis nicht fähig ist, es ist vielmehr ein menschliches Grundbedürfnis. Auch der Intellektuelle erstrebt „die Geborgenheit einer geordneten und sinnvollen Welt", und er ist durchaus bereit, dafür sogar „ein wenig von der Kritik zu opfern, die der gebildete Agnostiker für den Prüfstein seiner Intelli- 
genz und Freiheit hält. An Arthur Koestler, der trotz Einsicht in die Absurdität seines kommunistischen Glaubens lange und überlegt zögerte, diesen Glauben aufzugeben, ist dieses Bedürfnis nach einem bergenden und sinngebenden ideologischen System und die Furcht vor dem ideologischen Vakuum abzulesen. ${ }^{\circ 63}$ Besonders aufschlußreich ist auch Lembergs Hinweis, daß Ideologien immer mit Dichotomien arbeiten, insbesondere mit Freund-Feind-Klischees, und daß ihre Feindbilder durch das Fehlen jeglicher Differenzierung gekennzeichnet sind, weil „auf deren Einfachheit und Eindeutigkeit es mehr ankommt als auf ihren Wahrheitsgehalt" ${ }^{\text {"64. }}$. Und er meint, daß dies ,offenbar auf eine im menschlichen Denken angelegte Bipolarität" zurückzuführen sei ${ }^{65}$ - was denn auch den alten Gegensatz von naturalistischer und idealistischer Anthropologie erklären würde.

Natürlich können auch Ideologien in Frage gestellt und verändert werden. Sie müssen es allein schon deshalb, weil die Gesellschaft wie der einzelne, die sich zu ihnen bekennen, gezwungen sind, das ideologische System mit dem Fortschritt der Erkenntniswahrheit in Übereinstimmung zu halten. Aber nicht von ungefähr läßt sich eine Fülle von Beispielen dafür anführen, daß wissenschaftliche Erkenntnisse sich lange Zeit nicht durchsetzen konnten, weil ihnen der Anspruch der Ideologie entgegenstand. Offenbar sind also Einstellungsänderungen besonders dann schwer zu erreichen, wenn durch sie die Sicherheit und Geborgenheit des einmal gewonnenen Weltbildes gestört wird. Diese Geborgenheit aber wird wesentlich nur erreicht durch Vereinfachung.

Lemberg spricht von einem anthropologischen Dilemma: Einerseits ist eine vereinfachende Ideologie für die Erklärung der sonst unverständlichen Welt unentbehrlich, andererseits bedeutet Vereinfachung oft auch Verfälschung; insofern sind auch ideologische Systeme in Frage zu stellen, aber nichts fällt dem Menschen schwerer als das - eine für die Massenkommunikation grundlegende Tatsache.

\section{Schritte zur Konkretisierung}

Bisher war ständig nur von „dem Menschen“ die Rede. Einen solchen abstrakten Menschen jedoch gibt es nicht. Es gibt nur Menschen eines bestimmten Alters, einer bestimmten sozialen Herkunft, einer bestimmten Kultur, eines bestimmten Zeitalters. Es liegt auf der Hand, daß diese Faktoren für die Fernsehwirkungsforschung von größter Bedeutung sind. Im folgenden soll nur der erstgenannte herausgegriffen und thematisiert werden, die Tatsache also, daß der Mensch weder als Erwachsener noch als Fernsehzuschauer geboren wird, sondern dazu erst heranwächst. Für diese Genese aber sind die Altersstufen von Bedeutung, wenngleich festzuhalten ist, daß deren konkrete Ausgestaltung wiederum abhängig ist von einer Fülle äußerer, aber auch innerer Faktoren. Deren wechselseitige Abhängigkeiten zu fassen, macht ja das methodische Zentralproblem der empirischen Forschung aus. Insofern kann streng genommen ein Faktor gar nicht „herausgegriffen“, sondern nur im Kontext der jeweils anderen akzentuiert werden.

Der neugeborene Mensch muß sich die Welt, in die er hineingeboren ist, also eine jeweils bestimmte, technisch-kulturell-gesellschaftlich geprägte Welt erst erwerben und auf diese Weise zugleich das gesamte Inventar seiner Verhaltensweisen aufbauen: seines Wissens und Könnens, seiner Einstellungen und Wertungen, seiner Sprache und seines Denkens. Er kann aber nur dann verläßliche Verhaltensstrukturen aufbauen und so Verhaltenssicherheit gewinnen, wenn die Komplexität 
der Umwelt reduziert, also vereinfacht wird; dies um so mehr, je jünger ein Kind ist. Das geschieht durch die Eltern bzw. die Familie. Ihre Aufgabe ist es, eine stabile und konsistente, in sich widerspruchsfreie Umwelt zu schaffen; dies ist die Bedingung dafür, daß das Kind seinerseits in sich konsistente Verhaltensweisen aufbauen kann. „Wenn heute belohnt wird, was gestern bestraft wurde, dann entsteht Verhaltensunsicherheit. Die Welt erscheint chaotisch, unstrukturierbar, eben deshalb auch unlernbar; Apathie folgt daraus." 66

Ein wesentliches Element dieser Umweltkonsistenz ist auch die Stabilität der emotionalen Eltern-Kind-Beziehung. Diese emotionale Geborgenheit und Sicherheit, die vor allem rationalen Begreifen erfahren wird, „bewirkt die Bekräftigung des Kindes als das, was es ist". Erst die Erfahrung, daß es von den Eltern angenommen ist, ermöglicht ihm, sich selber anzunehmen, und erst so „kann es etwas aus sich machen. Fehlte diese Erfahrung, müßte vor allem Handeln immer die Angst stehen, sich selber aufs Spiel zu setzen." ${ }^{\text {67 }}$

Doch was den Vorzug der Familie in der frühkindlichen Lebensphase ausmacht, etwa in den ersten vier Jahren, kann zur Schwäche in späteren Lebensabschnitten werden. Manche Familien bieten dann zu wenig Anregungen, sind ,nicht komplex genug, um den wachsenden Differenzierungsbedürfnissen des Kindes elastisch folgen bzw. vorangehen zu können" 68 . Hier nun könnte der Bildschirm als Anreger wirken, die notwendigerweise einfachen, weil grundlegenden Verhaltensweisen des Kindes ausbauen und differenzieren helfen. Doch seine Möglichkeiten sind für das Vorschulkind gering. Zwar kann er ihm Wissenselemente vermitteln, bis hin zu Zahlen und Buchstaben, wie die Sesamstraße gezeigt hat, kaum aber neue Verhaltensweisen. Banal gesagt: das Kind kann, da es ja noch ganz in die Familie eingebunden ist, nur das an neuen Verhaltensweisen übernehmen, was die Eltern ihm zu übernehmen erlauben. Seine Lernmöglickeiten sind also rückgebunden an das familienpädagogische Gesamtklima ${ }^{69}$. Das ändert sich mit dem Schuleintritt und der damit verbundenen Ausweitung der Erfahrungsmöglichkeiten, grundlegend freilich erst im Durchgang durch die Pubertät, wenn die Ablösung von Eltern und Familie, die die Kehrseite der Verselbständigung darstellt, kräftig voranschreitet.

Bei Fernsehpraktikern stößt man häufig auf die Meinung, die Beschäftigung mit der Wirkungsforschung lohne sich nicht, da es zu jeder These eine Gegenthese gebe, und jede sei empirisch belegt. Dieser Eindruck stellt sich ein, solange man über empirische Einzeldaten und Teiltheorien nicht hinauskommt. Tatsächlich kann das Fernsehen beim Kind unterschiedliche, sogar entgegegesetzte Wirkungen haben. Es kann dem Eskapismus dienen, aber auch der Realitäts-Orientierung in der Erwachsenenwelt, es kann zur Nachahmung verleiten, diese aber auch blockieren. Ganz zu schweigen von den langfristigen oder gar den latenten Wirkungen, den stabilisierenden Einflüssen oder solchen, deren Möglichkeit noch gar nicht in Erwägung gezogen worden ist. Welche Wirkung tatsächlich eintritt, ist abhängig vom Rezipienten selbst, von seinen psychischen Dispositionen, seinem altersbedingten Entwicklungsstand, seinen familialen Gegebenheiten, dem sozialen und kulturellen Umfeld, aber auch von situativen Faktoren; oder in unserer bisher verwendeten Terminologie formuliert: sie ist abhängig von den jeweils aufgebauten Verhaltensstrukturen.

Es müßte also untersucht werden, welche Wirkungen unter welchen Bedingungen auftreten. Wenn ein Kind das Fernsehen als eskapistische Möglickeit nutzt, so hat es 
Gründe dafür, die aufgedeckt werden können, die freilich nicht einfach global mit dem Bedürfnisbegriff zu fassen sind. Gäbe es das Fernsehen nicht, so würde dieses Kind tagträumen oder andere Fluchtmöglichkeiten suchen. Ahmt ein Kind auf dem Bildschirm gesehene Gewalttätigkeit nach, ein anderes hingegen nicht, so haben beide Verhaltensweisen ebenfalls ihre Ursachen, die freilich wiederum nicht monokausal gefaßt werden können. Man weiß heute, „,daß das Kind um so mehrgegen die Beeinflussung durch ein Modell immun wird, je breiter gefächert seine Interessen sind ${ }^{670}$, je mehr eigene Vorstellungen und Handlungsperspektiven es also bereits erworben hat. Es kann aber auch sein, daß es die gesehene Gewalt nicht nachahmt, weil sie in ihm Angst erregt hat oder weil moralische Wertvorstellungen es daran hindern. Wenn diese Wertvorstellungen aggressives Handeln blockieren, so ist damit jedoch noch nichts über sein Denken gesagt. Wenn es hingegen umgekehrt aggressive Modelle nachahmt, so liegt das möglicherweise nur an seiner situativen emotionalen Verfassung.

Die knappen Hinweise sollen verdeutlichen, daß die sogenannten Fernsehwirkungen eine Vielzahl von Ursachen und Bedingungen haben können. Das ist gewiß keine neue Einsicht, sie scheint sich sogar zunehmend durchzusetzen, unsere These jedoch ist, daß sie vielfältigen Faktoren letzten Endes nur innerhalb eines umgreifenden anthropologischen Rahmens anzuordnen und einander zuzuordnen sind. Sonst bleibt über der Untersuchung einzelner psychischer und gesellschaftlicher Gesetzlichkeiten der anthropologische Zusammenhang auf der Strecke, man sieht den Wald vor lauter Bäumen nicht mehr oder die jeweils spezifischen Perspektiven verzerren das Ganze. Vor allem kommen jene Mechanismen und ihre anthropologische Bedeutung nicht mehr in den Blick, die für die ideologischen Systeme kennzeichnend sind.

Diese sind bereits für die Kinder von Bedeutung. Vermutlich ist dies sogar die zentrale Frage, wie die Plastizität der menschlichen Natur auch unter diesem Aspekt durch das Fernsehen mitgeformt wird. Einmal durch die formalen Wirkungen des Fernsehens, die H. v. Hentig aphoristisch so beschreibt: „Kindheit heute ist Fernsehkindheit: Die Welt erscheint verkleinert, zerstückelt, an- und abstellbar, in absurder Mischung, ohne Zusammenhang in sich und erst recht mit ihr. Dabei ist sie aufregend, extrem, glanzvoll und elend, übertrifft in allem meine kleine erlebbare Umwelt und macht sie unbedeutend."71 Zum anderen durch ihre inhaltlichen Wirkungen: Welche Weltanschauung, welche Sinnorientierung und Welterklärung vermittelt der Bildschirm bereits bei Kindern? Zwar ist deren Erwerb von Einstellungen und Verhaltensweisen rückgebunden an jene der Eltern, aber deren Einstellungen sind ja ebenso wie das familienpädagogische Gesamtklima bereits langfristig mitbeeinflußt durch das Fernsehen.

Von größter Bedeutung sind die den ideologischen Systemen eigenen Vereinfachungen für das Jugendalter. Keineswegs nur der Feindbildmechanismus. Der Jugendliche, der den „Schritt ins Leben“ tut und dabei dessen Kompliziertheit erfährt; der im Bemühen um Selbstbestimmung sich von bisherigen Bindungen und Autoritäten löst und sie unnachgiebig kritisiert - was das Kind noch nicht kann -, er braucht die vereinfachenden Ideologien, ,ihre vor allem beim Aufbau des moralischen Urteils erforderliche Hilfe" "72. Er klammert sich an die Unbedingtheit der Idee und der Gesinnung, verabscheut Kompromisse, kann eben deshalb freilich auch „Dinge unternehmen, zu denen er sich später nie wieder entschließen 
könnte"73. Lemberg meint, ,wer Gelegenheit hatte, mit jungen Menschen in einer Weise 'Geschichte zu betreiben', die ihre spontane Stellungnahme herausforderte, der kennt das leidenschaftliche Verlangen nach einem eindeutigen moralischen Urteil über die Vorgänge und Menschen, die die Geschichtsschreibung überliefert, einem Urteil, das möglicherweise in sein Gegenteil umschlägt, wenn der bisher bewunderte Held einer Handlung überführt wird, die im Moralkodex des Jugendlichen als negativ gilt"74. Der Jugendliche stelit also in Frage, Kritik kennzeichnet gerade dieses Lebensalter, aber seine Antworten sind unangemessene Vereinfachungen. Auch hier gilt wieder, daß solche umgreifenden anthropologischen Mechanismen wichtiger sein dürften als jene, die die Fernsehwirkungsforschung untersucht.

Der Erwachsene schließlich hat Standort und Selbstverständnis gefunden, grundsätzliche Neuorientierungen sind für ihn eher die Ausnahme als die Regel. Er verläßt sich auf das Erworbene, vor allem auf die erworbenen Grundorientierungen. Zwar muß auch er in der heutigen Zeit lebenslang lernen, aber sein Lernen ist vorwiegend ein Ausbauen und Differenzieren des Erworbenen, und wenn er Neues lernt, so muß auch dieses auf dem Vorhandenen aufbauen, mit ihm in Zusammenhang gesetzt werden. Auch er steht in der Grundspannung von Beharrung und Veränderung, aber das Beharrungsmoment ist ihm wichtiger. Manche Fernsehproduzenten möchten mit jeder Sendung etwas bewirken, am liebsten eine Meinungsund Einstellungsänderung. Aber das ist eine anthropologisch naive Vorstellung: Wollte der Zuschauer nach jeder Sendung seine Meinung ändern, gar noch zu den Grundfragen des Lebens, wollte er jedesmal einen Gesinnungswandel vollziehen, er käme, überspitzt gesagt, aus der Meinungs- und Gesinnungsrotation nicht mehr heraus. Selbst die Annahme ist bereits naiv, der Zuschauer, der täglich mehrere Stunden fernsieht, würde dies mit ununterbrochener konzentrierter Aufmerksamkeit tun.

Aus dem Gesagten ergibt sich eine Reihe von Konsequenzen. Zum Beispiel wird die Beliebtheit der populären Programme verständlich, über die die Kritiker nur die Nase zu rümpfen pflegen. G. Wiebe weist darauf hin, daß diese Beliebtheit historische Parallelen hat: „Mit Gutenbergs Erfindung der Buchdruckerkunst im 15. Jahrhundert waren auf einmal alle Bildungsschätze, die vorher strengen Beschränkungen unterlagen, zugänglich, soweit die Fähigkeit zu lesen reichte. Aber statt diese unverhoffte Gelegenheit zur Aufklärung zu ergreifen, verlangte das Publikum von Anfang an nur nach leichter, oberflächlicher und trivialer Lektüre. "75 Er erblickt den Grund für die Beliebtheit der „Unterhaltung“ darin, daß sie anthropologisch stabilisierend wirkt. Es sind jedem bekannte „gleichbleibende Grundsituationen und Grundmöglichkeiten“, so L. Bosshart ${ }^{76}$, die durchgespielt und miteinander kombiniert werden. Dabei können durchaus auch „Antiwerte“ dargestellt werden, etwa Respektlosigkeiten vor Autoritäten, aber auch sie wirken stabilisierend, insofern sie uns für Gehorsamsleistungen entschädigen, die wir im realen Leben nach wie vor aufzubringen bereit sind. Entscheidend ist, daß die vorgeführten Verhaltenstypisierungen und Rituale keine unliebsamen Überraschungen bergen. Zwar kann auch die Unterhaltung einzelne aufklärerische Elemente enthalten; sobald sich diese jedoch in den Vordergrund drängen, wird sie vom Publikum nicht mehr als Unterhaltung akzeptiert.

Es ist also unangebracht Unterhaltungsprogramme primär an ihrem „Realitätsgehalt" zu messen. Sie wollen gar keine Wirklichkeit vermitteln, auch keine nach- 
gebaute Wirklichkeit, sie entsprechen dem menschlichen Stabilitäts- und Beharrungsbedürfnis. Dieses aber ist nicht nur als notwendiges Übel in Kauf zu nehmen, man muß vielmehr seinen positiven anthropologischen Sinn sehen. Nun schlägt dieses Bedürfnis freilich auch durch gegenüber Informations- und Bildungssendungen, gegenüber den im weitesten Sinne „kritischen“ Programmen, jenen also, die in Frage stellen, die zur Auseinandersetzung mit Neuem und Anderem anregen wollen. Es erweist sich dann als Kommunikation blockierend. Praktiker wie Wissenschaftler der Kommunikation neigen dazu, dieses Phänomen nur negativ zu beurteilen; sie wollen dessen psychische und soziale Ursachen ergründen, um die Blockierungen dann nach Möglickeit auszuräumen. Man muß jedoch die Ambivalenz des Phänomens sehen.

In einer immer komplizierter werdenden Welt wachsen auch die Anforderungen an die Menschen, die in ihr leben. Es ist die Frage, ob nicht trotz der Plastizität der menschlichen Natur für manche diese Anforderungen bereits Überforderungen darstellen. Die wachsende Zahl derer, die „auf der Strecke bleiben“, physisch wie psychisch, scheint dafür zu sprechen. Ein Bestandteil dieser Welt ist nun aber auch das Fernsehen, das seine wichtigste Aufgabe darin erblickt, auch dem einfachen Bürger die. Welt weit über seinen Erfahrungsbe reich hinaus zugänglich zu machen. Täglich wird er mit weltweiten Problemen konfrontiert, und man erwartet von ihm, daß er sich mit ihnen auseinandersetzt, daß er das täglich Gebotene intellektuell und emotionell verarbeitet. Damit ist nichts gegen das Fernsehen gesagt, die wachsende Komplexität der Gesellschaft erfordert immer komplexere Kommunikationssysteme. „Der Übergang zur Hochkultur wird erst möglich durch die Entwicklung schriftlicher Kommunikation"; wer in der Hochkultur lebt und an ihr teilhaben will bzw. muß, kann nicht länger Analphabet sein. Für die heutige Welt gilt, „daß das soziale System der Weltgesellschaft für seine kommunikative Integration Massenmedien voraussetzt "77. Doch man muß auch die Kehrseite sehen und fragen, ob nicht der Durchschnittsmensch überfordert wird durch die rasante Veränderung in nahezu allen Lebensbereichen einerseits, durch die ständige durch Medien vermittelte Konfrontation mit weltweiten Veränderungen, Katastrophen, Problemen andererseits.

Zur Verdeutlichung könnte man auf das Streßphänomen hinweisen. Ursprünglich meinte H. Selye mit dem von ihm 1936 eingeführten Begriff Streß die physische Überbelastung, auf die der Körper mit „Alarmreaktionen“ antwortet. Inzwischen ist der Begriff zu einer psychosomatischen, sogar zu einer anthropologischen Kategorie geworden. Selye war nun aber nicht etwa der Meinung, man solle den Menschen möglichst vor jeglichem „Druck“, so die wörtliche Übersetzung, bewahren, er bezeichnet den Streß vielmehr zugleich als „Salz des Lebens“. Er hat also durchaus die Ambivalenz dieses Phänomens gesehen. Wenn heute der Begriff zur Kennzeichnung der extremen Belastungen benutzt wird, denen der Mensch der Gegenwart ausgesetzt ist, so läßt sich schwerlich leugnen, daß auch die Massenmedien ihren Teil zur Belastung bzw. Überbelastung beitragen, insbesondere dann, wenn sie problematisieren und in Frage stellen. Den unmittelbaren realen Belastungen kann man nur schwer entgehen, viel leichter hingegen ist es, sich jenem Druck zu entziehen, der nur ein von den Medien vermittelter ist. Der Fernsehzuschauer braucht nur ab- oder umzuschalten, im wörtlichen wie im übertragenen Sinn. Die reichlich angebotene Unterhaltung entlastet ihn auf die einfachste Weise, freilich nicht nur vom Druck der Arbeitswelt, sondern in einem viel umfassenderen Sinn. 
Das Gesagte gilt insbesondere für Sendungen, die nicht nur zur Auseinandersetzung anregen, sondern Meinungs- und Einstellungsänderungen bewirken wollen. Die Meinung über periphere Dinge kann man leicht und relativ folgenlos ändern, auf nichts aber ist man mehr angewiesen als auf die Verläßlichkeit seiner Grundeinstellungen, seiner grundsätzlichen Weltanschauung. Sie gibt dem Menschen zwar keine letzte Sicherheit, denn auch sie kann ihm fraglich (gemacht) werden, doch bildet sie die Basis seiner Welt- und Lebensorientierung. Das Fernsehen aber überschüttet ihn nicht nur mit einem Chaos von Informationen, in einer pluralistischen Gesellschaft wird er überdies ständig mit unterschiedlichen Weltanschauungen und Wertvorstellungen, mit dauernd wechselnden Parolen konfrontiert. Und nicht nur konfrontiert, er wird von ihnen umworben. Er würde hin und her gezerrt, wenn er sich zerren ließe.

Nun soll unsere Argumentation aber keinesfalls auf die Forderung hinauslaufen, das Fernsehen solle die Einstellungen des Publikums nur bestätigen. Das hieße, aus der Not des menschlichen Daseins eine Tugend zu machen, zugleich wäre es das Ende von Kommunikation. Es soll vielmehr die menschliche Grundspannung von Beharrung und Veränderung deutlich gemacht und aufgezeigt werden, daß auch die Tendenz zur Beharrung und damit zur Resistenz gegen Verunsicherungen einen „vernünftigen" Sinn hat. Auch die erworbenen Verhaltensweisen bedürfen der Bestätigung, um nicht wieder zu zerfallen, und sei es durch den Mechanismus der selbstbestätigenden Selektivität oder jenen, den Festinger als soziale Unterstützung bezeichnet. Die Tendenz des Menschen zur Beharrung dient gewissermaßen als Hilfe zur Erhaltung der Kontinuität seiner Persönlichkeit.

Zugleich soll unsere Argumentation verdeutlichen, daß das In-Frage-stellen ein schwieriges Geschäft ist, auch wenn ihm Neugier und Wissensdurst auf seiten der Rezipienten zumindest partiell entgegenkommen. Die berufsmäßigen Vermittler müssen die dafür nowendigen Voraussetzungen erwerben. Schon das Sprichwort sagt, daß ein Dummer mehr fragen kann, als zehn Weise zu beantworten in der Lage sind. Jeder Lehrer weiß, daß man dumme und kluge, pädagogische und unpädagogische, erhellende und verstellende, eindringliche und aufdringliche Fragen stellen kann. Fragen können zum Verhör mißraten. Sie können echt sein, d.h. der Fragende möchte tatsächlich etwas erfahren, sie können aber auch unecht sein; so ist das heute nicht zuletzt im Fernsehen modische „Hinterfragen" sehr oft gar kein wirkliches Fragen, sondern ein mehr oder minder geschickt getarnter Bekehrungsversuch. Eben dieses ist aufschlußreich und weist ins Zentrum unserer Argumentation zurück: Es sind die Positionen der anderen, die in Frage gestellt werden, selten oder nie die eigenen. Es zeigt sich also auf seiten der Kommunikatoren dasselbe Phänomen wie auf seiten der Rezipienten. Das ist zwar von dem hier vertretenen Ansatz her selbstverständlich, soll aber trotzdem erwähnt werden, denn es folgt daraus, daß alles bisher Ausgeführte nicht nur auf den Rezipienten des Fernsehens, sondern auch den Produzenten hin konkretisiert werden müßte.

Hier jedoch soll nur festgehalten werden, daß es unter dem Aspekt der Kommunikation weniger darauf ankommt, die positiven Einstellungen des Publikums in Frage zu stellen - diese sind im Gegenteil ausdrücklich zu achten -, als vielmehr darauf, zugleich die Einstellungen anderer gesellschaftlicher Gruppen verstehbar zu machen. In einer pluralistischen Gesellschaft mit ihren konkurrierenden Wertvorstellungen dürfte dies die zentrale kommunikative Aufgabe sein. Auch deshalb, 
weil nur so bei allen Unterschieden zugleich die Gemeinsamkeiten deutlich werden, die für die Existenz auch einer pluralistischen Gesellschaft unabdingbar sind. Tatsächlich jedoch ist in unseren Rundfunkanstalten die Zahl derer groß, die am Unfug der Polarisierung mitwirken statt zu seiner Überwindung beizutragen, die Kommunikation verhindern, statt sie zu ermöglichen, die es nicht dem Zuschauer überlassen, sich seine Meinung aufgrund solider Information selbst zu bilden, sondern jeweils gleich dazu sagen, was er von dem Berichteten zu halten habe.

Auf die damit auftauchende Frage nach einer Didaktik der Massenkommunikation ist hier nicht einzugehen, sie wäre eine eigene Abhandlung wert. Doch ist im $\mathrm{Zu}$ sammenhang unseres Themas der Hinweis angebracht, daß auch sie die anthropologischen Rahmenbedingungen von Kommunikation zu berücksichtigen hat ${ }^{78}$. Gewiß ist jede Sendung, selbst jede Nachrichtenmeldung ihrerseits bereits eine Redaktion von Komplexität; oder wie es K. Steinbuch unlängst in einer ZDFSendung formuliert hat, Journalisten sind Leute, deren Geschäft darin besteht, Probleme zu vereinfachen und sie so „telegen“ zu machen; aber das muß nicht den Verzicht auf Differenzierung bzw. differenzierende Strukturierung bedeuten, wie ein Blick auf die Theorie der Didaktik zeigt.

\section{Schlußbemerkungen}

Die vorliegenden Ausführungen sind sicher in vieler Hinsicht unzureichend. Zum einen müßten weitere Aspekte aufgegriffen werden, etwa die Frage „Warum sehen wir fern?“79; eine Anthropologie der menschlichen Sinnenhaftigkeit könnte zur Beantwortung dieser Frage aufschlußreiche Beiträge liefern ${ }^{80}$. Auch kulturanthropologische Aspekte müßten eingebracht werden. Zum anderen müßte vieles von dem hier Gesagten konkretisiert werden, vor allem müßten die vorliegenden Forschungsansätze, die sich teils überschneiden, teils nebeneinander, téils gegeneinander stehen, innerhalb des skizzierten Rahmens lokalisiert werden. Dies schon deshalb, damit nicht der falsche Eindruck entsteht, hier solle empirische Forschung durch philosophische „Spekulation“ ersetzt werden. Daß dies ein völliges Mißverständnis wäre, soll abschließend noch einmal betont werden. Hier ging es zunächst nur darum, eine umgreifende anthropologische Rahmentheorie zu skizzieren und sie wenigstens partiell zu konkretisieren. Die beiden verbleibenden Aufgaben, die Berücksichtigung weiterer Aspekte wie die systematische Einbeziehung vorhandener Teiltheorien, müßten eigens thematisiert werden.

Schließlich sei auch noch betont, daß unsere Argumentation keineswegs bloße Spekulation ist. Denn einmal stützt sie sich auf wesentliche Elemente abendländischen anthropologischen Denkens ebenso wie auf gegenwärtige anthropologische Thesen, etwa das letzte Buch Lembergs, das er als Abschluß seines Lebenswerks verstand. Zum anderen lassen sich durchaus auch empirische Belege für das Vorgetragene anführen. Dafür wenigstens ein Beispiel. Zu Beginn der 70er Jahre strahlte das Westdeutsche Fernsehen den Brückenkurs „Mathematisches Vorsemester“ aus. Das Autorenteam, junge Angehörige der Universität Bielefeld, wollte zeigen, daß man auch das Fach Mathematik „emanzipatorisch“ lehren könne und entwickelte zu diesem Zweck eine Präsentationsform, die man als Kritiker-Modell bezeichnete. Neben dem Fernsehlehrer, der den Stoff vermittelte, trat ein Kritiker auf, der die dargebotenen Inhalte und deren Didaktik problematisierte. Die Reaktion der Kursteilnehmer war enttäuschend. Sie lehnten die als „Anwalt der Zuschauer” 
intendierte Rolle des Kritikers ab. Er war also dem Lernprozeß nicht förderlich, sondern „löste Unsicherheit aus" ${ }^{\text {“81 }}$. Selbst bei einem relativ harmlosen Lernprozeß also zeigten sich jene anthropologischen Gegebenheiten, um die es uns ging.

Bei aller Hochschätzung der empirischen Forschung, deren Notwendigkeit nicht in Zweifel gezogen werden kann, sollte jedoch der Hinweis Browns nicht ignoriert werden: „Es stimmt, daß die sozialwissenschaftlichen Forschungsmethoden eine im wesentlichen schlecht zurechtgeflickte Erbschaft der Naturwissenschaften und eigentlich ungeeignet sind, den direkten menschlichen Erfahrungen mit der sozialen Wirklichkeit zu entsprechen. Dennoch ermöglichen sie es - zusammengenommen -, einige Aspekte der Vielschichtigkeit sozialen Verhaltens zu erkennen. Und zur Zeit steht uns nichts Besseres zur Verfügung. “82 Neben dieser methodischen Problematik der empirischen Sozialforschung ist überdies die Grundversuchung der Empirie zu beachten, nämlich nur das Meßbare für real zu halten.

\section{Anmerkungen}

1 H. Sturm: Einführung zu: Fernsehen und Sozialisationsprozesse in der Familie. In: „Fernsehen und Bildung" 9:1975, S. 97.

${ }^{2}$ U. Saxer: Forschungen im deutschsprachigen Raum zum Thema Fernsehen und Sozialisationsprozesse in der Familie. In: „Fernsehen und Bildung“ 9:1975, S. 188.

${ }^{3}$ G. Tulodziecki (Hrsg.): Schulfernsehen in der Bundesrepublik Deutschland. Köln 1977, S. 162 f.

${ }^{4}$ Ebda., S. 89.

5 J. Hackforth: Massenmedien und ihre Wirkungen. Göttingen 1976, S. 184.

6 U. Saxer: Medienverhalten und Wissensstand - Zur Hypothese der wachsenden Wissenskluft. In: Buch und Lesen, bertelsmann texte 7, Gütersloh 1978, S. 42.

${ }^{7}$ So etwa J. Hackforth: Wirkungsforschung ja, aber anders! In: Bertelsmann Briefe Heft 92, 1977, S. 21 - 26. H.-D. Kübler: Zur Kritik der Kinder-Fernsehforschung oder ein Paradigma für die fällige Revision der Medienforschung. In: Bertelsmann Briefe Heft 101, 1980, S. 3 14. K. Renckstorf: Neue Perspektiven in der Massenkommunikationsforschung. Beiträge zur Begründung eines alternativen Forschungsansatzes. Berlin 1977. Aber auch P. Hunziker, H. Kellner, W. Teichert und weitere können genannt werden.

${ }^{8}$ Th. Kuhn: Die Struktur wissenschaftlicher Revolution. Frankfurt/M. 1973.

${ }^{9}$ So K. Renckstorf, W. Teichert u.a.

${ }^{10} \mathrm{Vgl}$. U. Saxer: Medienverhalten und Wissensstand, a.a.O.

11 H.-D. Kübler: Zur Kritik der Kinder-Fernsehforschung . ., a.a.O., S. 11.

12 Ebda., S. 10.

13 Vgl. etwa D. McQuail: Kommunikationsmodelle und Fernseheinfluß. In: H. Sturm/J.R. Brown (Hrsg.): Wie Kinder mit dem Fernsehen umgehen. Stuttgart 1979, S. 307-324.

14 J. R. Brown: Einführung zu H. Sturm/J.R. Brown (Hrsg.), a.a.O., S. 11.

15 Ebda., S. 111 .

16 Ebda., S. 17.

17 Vgl. A. Portmann: Das Tier als sozjales Wesen. Zürich 1953.

18 R. Dahrendorf: Homo Sociologicus. Köln/Opladen ${ }^{10} 1971$, S. 17 f. u.ö.

19 J. Kob: Soziologische Theorie der Erziehung. Stuttgart 1976, S. $17,19$. 
N. Luhmann: System - Systemtheorie. In: Ch. Wulf(Hrsg.): Wörterbuch der Erziehung. München 1974, S. 584.

21 F. v. Cube: Kybernetik und Pädagogik. In: Ch. Wulf (Hrsg.): a.a.O., S. 364.

$22 \mathrm{Vgl}$. L. v. Bertalanffy: General system theory: Foundations, development, applications. London 1971.

23 Vgl. E. König/H. Riedel: Systemtheoretische Didaktik. Weinheim 1973.

24 N. Luhmann: a.a.O., S. 583.

25 N. Luhmann: Moderne Systemtheorie als Form gesamtgesellchaftlicher Analyse. In: J. Habermas/N. Luhmann: Theorie der Gesellschaft oder Sozialtechnologie - Was leistet die Systemtheorie. Frankfurt/M 1971, S. 12.

26 N. Luhmann: System - Systemtheorie, a.a.O., S. 584.

27 M. Irle/V. Möntmann: Die Theorie der kognitiven Dissonanz: Ein Resümee ihrer theoretischen Entwicklung und empirischen Ergebnisse 1957-1976. In: L. Festinger: Theorie der kognitiven Dissonanz, hrsg. v. M. Irle/V. Möntmann. Bern 1978, S. 277.

28 Vgl. ebda., S. $277 \mathrm{ff}$.

29 J. Hackforth: Massenmedien und ihre Wirkungen, S. $22 \mathrm{ff}$.

30 Ebda., S. 23.

31 Vgl. B. Bossong: Motivationsförderung in der Schule. Weinheim 1978.

32 L. Festinger: a.a.O., S. 267 f.

33 Ebda., S. 15.

34 Ebda., S. 22f.

35 Ebda., S. 28-30.

36 Vgl. ebda., S. 256.

37 Vgl. ebda., S. 259-263.

38 G. Maletzke: Zur wissenschaftlichen Erforschung des Fernsehens. In: G. Maletzke (Hrsg.): Einführung in die Massenkommunikationsforschung. Berlin 1972, S. 154f.

$39 \mathrm{Vgl}$. die Zusammenstellung der Kritikpunkte bei J. Hackforth: a.a.O., S. 34ff.

$40 \mathrm{Vgl}$. u.a. H. Sturm/S. Jörg: Informationsverarbeitung durch Kinder. Piagets Entwicklungstheorie auf Hörfunk und Fernsehen angewandt. Schriftenreihe Internationales Zentralinstitut für das Jugend- und Bildungsfernsehen Nr. 12. München 1980.

41 R. Fatke: Jean Piaget. In: H. Scheuerl (Hrsg.): Klassiker der Pädagogik II. München 1979 , S. 301 .

42 J. Piaget/B. Inhelder: Die Psychologie des Kindes. Olten 1972, S. 157. Hier zitiert nach R. Fatke: a.a.O., S. 307.

43 K. Pawek: Das optische Zeitalter. Olten 1963, S. 218.

44 Im folgenden wird z.T. aufGedankengänge zurückgegriffen, die bereits in dem Aufsatz des Verf: Macht und Ohnmacht der Massenmedien formuliert wurden. In: „Stimmen der Zeit“" 195:1977, S. 751ff.

45 Vgl. J. Derbolav: Systematische Perspektiven der Pädagogik. Heidelberg 1971, S. 55f.

46 Vgl. R. Merkert: Die Sinne des Menschen im Dienst des geistigen Welterwerbs. Wuppertal/Kastellaun/Ratingen 1970, S. 33f.

47 A. Gehlen: Anthropologische Forschung. Reinbek bei Hamburg 1961, S. 18

48 Vgl. A. Gehlen: Der Mensch - Seine Natur und seine Stellung in der Welt. Bonn ${ }^{6} 1950$, S. 176. 
49 A. Gehlen: Anthropologische Forschung, S. 49.

50 Ebda., S. 71.

51 Vgl. etwa E. Groß: Einführung in die Bildungssoziologie Band 1. Bad Heilbrunn 1975, S. $30 \mathrm{r}$.

52 So J. Kob: a.a.O., S. 18.

53 A. Gehlen: a.a.O., S. 72.

54 1. Bock: Kommunikation und Erziehung. Darmstadt 1978, S. 96.

55 J. Derbolav: a.a.O., S. 57.

56 Vgl. A. Gehlen: Der Mensch, S. 185.

$57 \mathrm{M}$. Pagnol hat dieses Grundphänomen menschlicher Kommunikation sehr plastisch veranschaulicht, freilich ohne dabei an das Fernsehen zu denken. Er erzählt, wie er sich von seinen Freunden feiern lie $\beta$, als er sein erstes Gedicht geschrieben hatte, und fährt dann fort: „Trotzdem verstehe und entschuldige ich diese lächerliche Eitelkeit eines 'Dichters von dreizehn Jahren, da ich seither eine ziemlich große Anzahl von Herren und Damen kennengelernt habe, die lange nach überstandener Pubertät mit Leidenschaft Oden, Sonette und sogar epische Gedichte verfassen. Ihre Gefühle sind echt und ihr poetischer Stil unmittelbar; sie haben eben die schönen Seelen der Dichter. Lesen sie uns ihre Werke vor, vergießen sie Ströme von Tränen, denn sie empfinden noch einmal die Gemütsbewegung, die sie inspiriert hatte und die sie glaubten in Worte fassen zu müssen. Der eine erzählt von Françoise, und die zweieinhalb Silben enthalten die erste Liebe seiner Jugend; er sagt 'Heuschrecke' und hört aus weiter Ferne die Musik des ersten Ferienabends; mit Inbrunst nennt er das Wort 'Abendgebet' und sieht die kleine, ländliche Kirche wieder vor sich, schlecht beleuchtet an einem Winterabend, als er neben seiner geliebten Mutter kniete. Aber da der Zuhörer die richtigen Schlüsse zu diesen Worten nicht hat, gebraucht er oftmals andere. Er ist nie zur Vesper in die Kirche gegangen. 'Heuschrecken' erinnern ihn an einen großen, starken Neger, der sie auf der Herdplatte röstete und darauf bestand, daß er wenigstens eine davon verzehrte, und Françoise ist für ihn der Name einer schielenden Köchin, die sich, als sie endlich entlassen wurde, rühmte, tagtäglich in die Suppe gespuckt zu haben. Darum ist das alles für den Zuhörer nur eine heruntergeschnurrte Litanei von Wörtern, und die Gemütsbewegung der Vortragenden erscheint ihm in jämmerlicher Weise unbegreiflich.“In: Die Zeit der Liebe. Goldmann-TB 3878, München 1980, S. 159 f.

58 U. Saxer: Führt ein Mehrangebot an Programmen zu selektivem Rezipientenverhalten? In: „Media Perspektiven“ 6/80, S. 395 ff.

59 E. Lemberg: Anthropologie der ideologischen Systeme. Weinheim 1977, S. 3. - Vgl. zu diesem Abschnitt auch das umfangreichere Werk E. Lembergs: ldeologie und Gesellschaft. Stuttgart 1971.

60 Ebda., S. 19.

${ }^{61}$ Ders.: ldeologie und Gesellschaft, S. 39.

62 Ders.: Anthropologie der ideologischen Systeme, S. IX,

63 Ebda., S. 119.

Eda., S. 78.

Ebda., S. 88.

Weidhardt: Strukturbedingungen und Probleme familialer Sozialisation. In: D. Claessens/ P. Milhoffer (Hrsg.): Familiensoziologie. Frankfurt/M 1973, S. 206.

Ebda., S. 211.

Eda., S. 230. 
69 Ist dieses anregungsreich, werden auch die Anregungen des Fernsehens verarbeitet. Denen, die haben, wird also noch dazu gegeben; denen, die nicht haben, wird auch das Wenige noch genommen - das ist der Kern der neuen These von der Zunahme der Wissenskluft durch das Fernsehen.

70 B. H. Kniveton: Soziales Lernen und Nachahmung. In: H. Sturm/J.R. Brown (Hrsg.): Wie Kinder mit dem Fernsehen umgehen. S. 279.

71 H. v. Hentig: Vorwort zu Ph. Ariès: Geschichte der Kindheit. dtv-Taschenbuch 4320, München 1978, S. 20.

72 E. Lemberg: a.a.O., S. 144.

73 R. Guardini: Die Lebensalter - Ihre ethische und pädagogische Bedeutung. Würzburg o.J., S. 22.

74 E. Lemberg: a.a.O., S. 140.

75 G. Wiebe: Zwei psychologische Faktoren im Verhalten des Medienpublikums. In: D. Prokop (Hrsg.): Massenkommunikationsforschung Bd. 2. Frankfurt/M 1973, S. 355.

76 L. Bosshart: Dynamik der Fernsehunterhaltung. Eine kommunikationswissenschaftliche Analyse und Synthese. Freiburg (Schweiz) 1979, S. 39.

$77 \mathrm{~N}$. Luhmann: Veränderungen im System gesellschaftlicher Kommunikation und die Massenmedien. In: O. Schatz (Hrsg.): Die elektronische Revolution. Graz/Köln 1975, S. 16ff.

78 Praktiker haben inzwischen einschlägige Faustregeln entwickelt, etwa: Wenn man Ungewohntes bringen wolle, dürfen nicht Inhalt und Form gleichzeitig ungewöhnlich sein. Wer z.B. Verständnis für eine bestimmte diskriminierte Minderheit wecken wolle, tue dies am besten innerhalb altvertrauter Formen, etwa eines Krimis: der Homosexuelle als trotzdem sympathischer und erfolgreicher Polizist; wer neue Formen erproben wolle, wähle möglichst vertraute Inhalte.

79 J.R. Brown: a.a.O., S. I1

80 Vgl. etwa E. Straus: Vom Sinn der Sinne. Berlin/Göttingen/Heidelberg ${ }^{2} 1956$.

81 R. Terlinden: Bildungsverständnis und Dritte Fernsehprogramme. Bochum 1979, S. 109. - Vgl. auch W. Löding u.a.: Mathematisches Vorsemester. Protokoll eines Experimentes. Bonn 1972.

82 J.R. Brown: a.a.O., S. 17. 


\section{SUMMARY}

Currently some authors speak of a certain sterility in research on the effectiveness of media. They look for a more adequate theory. In this contribution the author holds that only within a more basic anthropological framework would it be possible to integrate different results of research and show new productive questions. The first part of the thesis is supported by other partial and universal theories. Then a new attempt at an anthropological theory is introduced, starting from the two interpretations of human "questionability" and the tension between stability and change. The third and fourth sections deal with the consequences for the TV consumer, and an attempt is made to concretise these arguments.

\section{RÉSUMÉ}

Différents auteurs parlent, actuellement, d'une certaine stérilité de la recherche sur l'efficacité des medias. Ils exigent des rudiments de théories plus adéquats. Dans ce travail, on défend la thèse qu'en dernier lieu, seule une théorie-cadre anthropologique convient à intégrer les résultats des recherches, ceux existants et quelquefois contradictoires, et à rendre visibles également de nouveaux termes productifs du problème. Dans la première partie, la thèse est justifiée en accord avec des théories les unes trop partielles, les autres trop universelles. Puis, est esquissé un rudiment anthropologique de théories, partant d'une équivoque sur le "caractère problèmatique" humain et de la tension humaine fondamentale de persévérance et de changement qui en résulte. Dans la troisième et la quatrième partie, leurs conséquences sur le comportement des téléspectateurs sont mises en évidence, et on essaie de concrêtiser l'argumentation en quelques points, de façon rudimentaire.

\section{RESUMEN}

Diversos autores hablan en la actualidad de una cierta esterilidad en la investigación de la eficacia de los medios de comunicación social. Urgen una más adecuada teoría. En este trabajo se defiende la tesis de que, en última instancia, solo una teoría global antropológica podría integrar los contradictorios resultados de la investigación y sacar a la luz nuevos interrogantes fecundos. En la primera parte se justifica esta tesis confrontándola en parte con teorías parciales, en parte con teorias universales. Después se describe una teoría antropológica partiendo del doble sentido de la «incertidumbre» humana y de la resultante tensión fundamental de persistencia y cambio. En la tercera y cuarta parte se deducen sus consecuencias para el comportamiento del tele-espectador y se intenta concretar la argumentación en algunos puntos. 